\title{
Gravitational lensing by a quantum deformed Schwarzschild black hole
}

\author{
$\mathbf{X u ~ L u}{ }^{1,2}, \mathbf{Y i ~ X i e}^{2,1, \mathrm{a}_{\mathbb{D}}}$ \\ ${ }^{1}$ School of Astronomy and Space Science, Nanjing University, Nanjing 210023, China \\ ${ }^{2}$ Key Laboratory of Modern Astronomy and Astrophysics, Nanjing University, Ministry of Education, Nanjing 210023, China
}

Received: 4 May 2021 / Accepted: 10 July 2021 / Published online: 18 July 2021

(C) The Author(s) 2021

\begin{abstract}
We investigate the weak and strong deflection gravitational lensing by a quantum deformed Schwarzschild black hole and find their observables. These lensing observables are evaluated and the detectability of the quantum deformation is assessed, after assuming the supermassive black holes Sgr A* and M87* respectively in the Galactic Center and at the center of M87 as the lenses. We also intensively compare these findings with those of a renormalization group improved Schwarzschild black hole and an asymptotically safe black hole. We find that, among these black holes, it is most likely to test the quantum deformed Schwarzschild black hole via its weak deflection lensing observables in the foreseen future.
\end{abstract}

\section{Introduction}

Although Einstein's general relativity (GR) has been tested and verified in many experiments [1], it is commonly believed that GR is incomplete. A black hole is the simplest object in the universe predicted by GR. However, it contains a central singularity at which GR is invalid and an event horizon which might cause information paradox. Therefore, with extremely strong gravitational field, a black hole also provides an ideal and unique laboratory for examining GR and alternative theories of gravitation, which can access neither on the Earth nor in the Solar System. Direct imaging of the supermassive black hole M87* in the center of the giant elliptical galaxy M87 [2-7] and successful detection of the gravitational waves from the black holes merger events [8-13] demonstrate that black holes spread widely across the universe, paving the way for strong-field tests of new theories of gravitation which might cure the "illness" of a black hole.

In order to fix these problems once for all, we have to wait for a self-consistent and well accepted quantum the-

\footnotetext{
a e-mail: yixie@nju.edu.cn (corresponding author)
}

ory of gravity available. However, before that, there are still some effective ways to manifest the quantum effects on the spacetime of a black hole for removing the singularity, such as replacing it with a regular core [14-17], making use of quantum pressure to create a bouncing geometry [18-20] and formation of quasi black holes [21-24] (see Ref. [25] for a review). The event horizon could also be erased, if either an exotic compact object [26] or a compact quantum object [27] are considered. These horizonless objects are more or less involved with quantum dynamics and effects (see Ref. [28] for a review). Quantum characteristics and nature of the spacetime and gravitation have been widely and intensively investigated as well in various contexts and inspired by plenty of motivations [29-36]. They lead to quantum black hole solutions, such as those in the effective field theory [3739], in the asymptotically safe gravity [40-43], in the loop quantum gravity $[44,45]$, in the non-commutative geometry [46-49], and under the modified uncertainty principles [50,51].

In this work, we focus on the quantum deformed Schwarzschild black hole, which is affected by the spherically symmetric quantum fluctuations of the metric and the matter fields [52]. Its singularity shifts to a finite distance and becomes a divergent sphere, which connects two asymptotically flat world sheets. This is very different from the central singularity of the Schwarzschild black hole. These intriguing quantum behaviors have drawn much attention. The entropy of the quantum deformed Schwarzschild black hole with electrical charges [53], its quasinormal modes [54], its thermal dynamics and equation of state in the quintessence field [55], the tunneling process [56] and its unique phase transitions [57] were well studied. The quasinormal modes and grey-body factors of fields of various spin for the quantum deformed Schwarzschild black hole were analyzed through the WKB approach and time-domain integration method, and it was shown that the quantum deformation can cause the shadow cast by this black hole to decrease [58]. Its photon 
rings and lensing rings were also studied and the lensing ring was found to be brighter than the one of the Schwarzschild black hole [59]. However, the gravitational-lensing signatures of the quantum deformed Schwarzschild black hole are barely known, which requires a comprehensive investigation.

In the gravitational lensing, the trajectory of a photon propagating through the gravitational field of a massive body might be deflected and the arrival time of the photon might be delayed, providing insight on the nature of the spacetime [60]. The gravitational lensing might have two important scenarios according to its deflection angle. In the weak deflection gravitational lensing, the deflection angle is much smaller than 1 . It has been a invaluable tool in astronomy [61-64] and been widely employed in gravitational physics [65-69]. In the strong deflection gravitational lensing by a compact object, the photon passes its vicinity in the strong field so that its deflection angle might be much bigger than 1 . This will allow photons to loop around such an object by several times and to produce relativistic images [70]. The direct image of M87* by EHT [2-7] demonstrates the possibility of observing the strong deflection gravitational lensing by black holes, which would be helpful for understanding the physical properties of the black holes [71-78], for probing and distinguishing various kinds of black holes [79-84] and horizonless compact objects [85-101].

Gravitational lensing has also been widely adopted for detecting and testing the quantum nature of spacetime. The deflection angle in the weak deflection gravitational lensing might be affected by the quantum properties of some black holes in the effective field theory [102], in the asymptotically safe gravity [103] and with the vacuum effect $[104,105]$ so that the resulting lensing observables might be altered [106]. The shadow and other observables in the strong deflection gravitational lensing might also be inflected for some black holes in the asymptotically safe gravity $[107,108]$, in the loop quantum gravity [109] and in the non-commutative geometry [110-112]. In order to obtain a full understanding of the quantum effects, both weak and strong deflection gravitational lensing have been studied for some black holes in the asymptotically safe gravity $[113,114]$, in the loop quantum gravity [115], under the Extended Uncertainty Principle [116] and in the rainbow gravity [117].

Inspired by these previous works and hoping to gain a whole picture of the weak and strong deflection gravitational lensing [118-124], we will investigate these two scenarios of gravitational lensing by the quantum deformed Schwarzschild black hole for detecting and searching its quantum signatures. We will carefully compare its observables with those of two other quantum black holes, the renormalization group improved Schwarzschild black hole [41] and the asymptotically safe black hole [107], both of which were proposed in the asymptotic safety scenario in the quantum gravity and which have very similar spacetime metrics.
Although the weak and strong deflection gravitational lensing by the renormalization group improved Schwarzschild black hole was known [113], most lensing observables in the weak and strong deflection gravitational lensing by the asymptotically safe black hole are still unknown except its shadow [107] so that we will also fill in the blanks here. For the gravitational lensing by these quantum black holes, their observability in practice will be intensively discussed.

This paper is structured as follows. In Sect. 2, we brief the spacetime of the quantum deformed Schwarzschild black hole and the essentials of the gravitational lensing. In Sects. 3 and 4 , its weak and strong deflection gravitational lensing are investigated, respectively. The lensing observables and their observability are found and discussed by taking Sgr A* and M87* as lenses (if applicable). Conclusions and discussion are given in Sect. 5 .

\section{Spacetime and gravitational lensing}

\subsection{Metric}

Affected by the spherically symmetric quantum fluctuations of the metric and the matter fields, the spacetime of a quantum deformed Schwarzschild black hole with mass $m_{\bullet}$ was found as $(G=c=1)[52]$

$$
\mathrm{d} s^{2}=-A(r) \mathrm{d} t^{2}+B(r) \mathrm{d} r^{2}+C(r)\left(\mathrm{d} \theta^{2}+\sin ^{2} \mathrm{~d} \phi^{2}\right),
$$

with

$$
\begin{aligned}
& A(r)=[B(r)]^{-1}=-\frac{2 m_{\bullet}}{r}+\frac{\sqrt{r^{2}-a_{\bullet}^{2}}}{r}, \\
& C(r)=r^{2}
\end{aligned}
$$

where $a_{\bullet}$ controls the deformation originated from the effective two-dimensional dilaton gravity. When the quantum deformation vanishes, i.e., $a_{\bullet}=0$, the metric (1) reduces to the one of the Schwarzschild black hole. The real-valued metric demands a obvious limit on the radial coordinate that $r \geq a_{\text {e }}$. While such a spacetime is asymptotically flat as $r \rightarrow+\infty$, the quantum fluctuations make it not Ricci-flat, differing from the Schwarzschild black hole [52]. Its singularity where the curvature is infinite is located at $r=a_{\bullet}$ [52], which is always covered by the event horizon at

$r_{\mathrm{H}}=\sqrt{4 m_{\bullet}^{2}+a_{\bullet}^{2}}$.

When $a_{\bullet}=0, r_{\mathrm{H}}$ returns to the Schwarzschild one $2 m_{\bullet}$.

For later convenience, we define the following dimensionless variables

$x=\frac{r}{m_{\bullet}} \quad$ and $\quad x_{\mathrm{H}}=\frac{r_{\mathrm{H}}}{m_{\bullet}}$, 


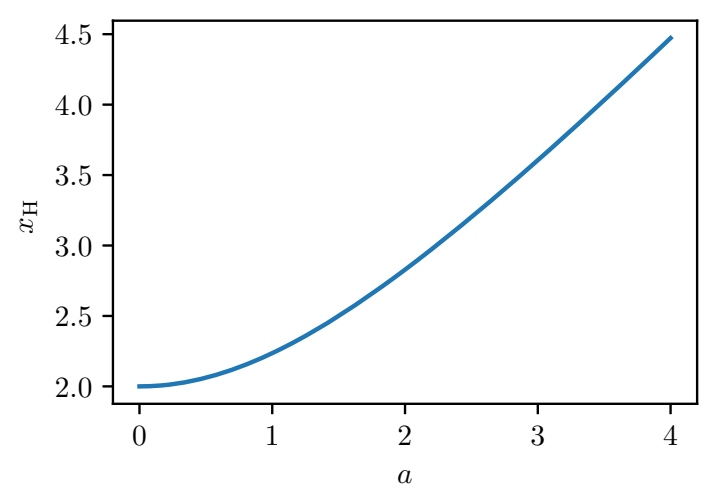

Fig. 1 The (dimensionless) radius of the event horizon for the quantum deformed black hole changes with the (dimensionless) deformation parameter

and the dimensionless deformation parameter

$a=\frac{a_{\bullet}}{m_{\bullet}}$,

so that the dimensionless radius of the event horizon is

$x_{\mathrm{H}}=\sqrt{4+a^{2}}$.

Figure 1 shows $x_{\mathrm{H}}$ with respect to $a$, indicating that the stronger is the quantum deformation, the bigger is the event horizon than the Schwarzschild one. It makes the quantum deformed Schwarzschild black hole quite different from other quantum-corrected black holes, such as the renormalization group improved Schwarzschild black hole [41] and the asymptotically safe black hole [107], that the quantum effects cause their event horizons to shrink.

As long as a photon stays outside the event horizon, it can freely propagate in the spacetime (1) with any possible real $a_{\bullet}$. However, it does not mean that $a_{\bullet}$ can be arbitrarily big, since it would significantly change the angular size of the shadow cast by the quantum deformed Schwarzschild black hole. Based on the direct image of the supermassive black hole M87* [2-7], it was found [125] that the angular size of the M87*'s shadow, which is consistent within $17 \%$ at $68 \%$ confidence level with the predicted size of GR, can be employed for various gravitational tests in the strong-field regime. Following the approach of Ref. [125], we obtain a preliminary bound on the (dimensionless) deformation parameter as (see Sect. 4.1 for details)

$\mathcal{D}=\{a \mid 0<a<1.53\}$,

which will be adopted in the following investigation on the weak and strong deflection gravitational lensing by the quantum deformed Schwarzschild black hole.

\subsection{Essentials of gravitational lensing}

In the asymptotically flat and spherically symmetric spacetime (1), the deflection angle of a photon $\hat{\alpha}$ can be determined by [126]

$\hat{\alpha}\left(r_{0}\right)=2 \int_{r_{0}}^{\infty} \frac{\mathrm{d} r}{r^{2} \sqrt{u^{-2}-A(r) r^{-2}}}$,

where $r_{0}$ is the closed approach between the photon and the black hole, $u$ is the impact parameter

$u=\frac{r_{0}}{\sqrt{A\left(r_{0}\right)}}$,

and the relation $A(r) B(r)=1$ has been used, see Eq. (2). In the weak deflection gravitational lensing that $r_{0} \gg m_{\bullet}$, the deflection angle $\hat{\alpha}$ will be much smaller than 1 . In the strong deflection gravitational lensing, $r_{0}$ is be close to $m$. and, thus, $\hat{\alpha}$ will diverge.

In this work, we adopt the following lensing equation to describe the geometric relation among the source, the lens and the observer as [71,72]

$$
\tan \mathcal{B}=\tan \vartheta-\frac{D_{\mathrm{LS}}}{D_{\mathrm{OS}}}[\tan \vartheta+\tan (\hat{\alpha}-\vartheta)],
$$

where $\mathcal{B}$ and $\vartheta$ are, respectively, the angular positions of the source and the lensing images, and $D_{\mathrm{LS}}$ and $D_{\mathrm{OS}}$ are, respectively, the distances from the source to the lens and to the observer. The magnification of the image $\mu$ is the flux ratio of the lensed image to the unlensed source, which is defined as [127]

$\mu=\left(\frac{\sin \mathcal{B}}{\sin \vartheta} \frac{\mathrm{d} \mathcal{B}}{\mathrm{d} \vartheta}\right)^{-1}$

If the source has time-evolved emission, the time delay between the two lensed images will be an important observable as well, which depends on the travel time of the photon from the source to the observer [126]

$T=T\left(R_{\mathrm{src}}\right)+T\left(R_{\mathrm{obs}}\right)$.

Here, $R_{\mathrm{src}}$ and $R_{\mathrm{obs}}$ are, respectively, the radial coordinates of the source and the observer relative to the lens that

$$
\begin{aligned}
R_{\mathrm{src}} & =\sqrt{D_{\mathrm{LS}}^{2}+D_{\mathrm{OS}}^{2} \tan ^{2} \mathcal{B}}, \\
R_{\mathrm{obs}} & =D_{\mathrm{OL}},
\end{aligned}
$$

where $D_{\mathrm{OL}}$ is the distance from the observer to the lens. The function of $T(R)$ is defined as

$T(R)=\int_{r_{0}}^{R}\left|\frac{\mathrm{d} t}{\mathrm{~d} r}\right| \mathrm{d} r$,

and it can be evaluated by the metric (1) as

$$
T(R)=\int_{r_{0}}^{R} \frac{\mathrm{d} r}{A(r) \sqrt{1-u^{2} r^{-2} A(r)}},
$$


where the relation $A(r) B(r)=1$ has been used.

In the following sections, the observables in both weak and strong deflection gravitational lensing will be investigated. Considering the routinely monitoring on $\mathrm{Sgr} \mathrm{A}^{*}$ by the ground-based telescopes in the optical/near-infrared band and the direct image of M87* by EHT in the radio band, we will take these supermassive black holes as the lenses (if applicable) for assessment of the lensing observables. In the meantime, we will carefully compare these observables with those of other quantum-corrected black holes, such as the renormalization group improved Schwarzschild black hole [41] and the asymptotically safe black hole [107], and intensively discuss their observability in practice.

\section{Weak deflection gravitational lensing}

In the weak deflection gravitational lensing, while the deflection angle of the trajectory of a photon is very small, it can affect the positions and magnification of the lensed images as well as the arrival time of the photon.

\subsection{Deflection angle}

In the scenario of the weak deflection gravitational lensing, the closet approach between the photon and the quantum deformed Schwarzschild black hole $r_{0}$ is much larger then the gravitational radius of the black hole $\sim m_{\bullet}$, so that $m_{\bullet} r^{-1}$ can be treated as a small parameter for any given $r \geq r_{0}$ of a photon, and $A(r)$ and $B(r)$ can be expanded in terms of it as

$$
\begin{aligned}
A(r)= & 1-2 \frac{m_{\bullet}}{r}-\frac{a^{2}}{2} \frac{m_{\bullet}^{2}}{r^{2}}-\frac{a^{4}}{8} \frac{m_{\bullet}^{4}}{r^{4}}+\mathcal{O}\left(\frac{m_{\bullet}^{5}}{r^{5}}\right) \\
B(r)= & 1+2 \frac{m_{\bullet}}{r}+\left(\frac{a^{2}}{2}+4\right) \frac{m_{\bullet}^{2}}{r^{2}}+\left(2 a^{2}+8\right) \frac{m_{\bullet}^{3}}{r^{3}} \\
& +\left(\frac{3}{8} a^{4}+6 a^{2}+16\right) \frac{m_{\bullet}^{4}}{r^{4}}+\mathcal{O}\left(\frac{m_{\bullet}^{5}}{r^{5}}\right) .
\end{aligned}
$$

We can see that the quantum deformation manifests itself starting from the second-order term of $m_{\bullet} r^{-1}$.

Before we proceed, it is necessary to compare these expanded forms with those of the renormalization group improved Schwarzschild black hole [41] and the asymptotically safe black hole [107], which are given in Appendix A. The quantum improvement for the renormalization group improved Schwarzschild black hole is dominantly controlled by the parameter $\Omega$, while the quantum correction for the asymptotically safe black hole is characterized by the parameter $\Gamma$. By looking at Eqs. (18) and (19) for the quantum deformed Schwarzschild black hole, Eqs. (A.3) and (A.4) for the renormalization group improved Schwarzschild black hole, and Eqs. (A.7) and (A.8) for the asymptotically safe black hole, we find that the quantum effects of $a, \Omega$ and $\Gamma$ begin to play their roles, respectively, from the second-, third- and fourth-order terms of $m_{\bullet} r^{-1}$ in their expanded metrics. Hence, we can expect that these quantum effects in the weak deflection gravitational lensing by the quantum deformed Schwarzschild black hole, the renormalization group improved Schwarzschild black hole and the asymptotically safe black hole will orderly decrease. As a result, the quantum deformed Schwarzschild black hole is most likely to be detected by the weak deflection gravitational lensing, followed by the renormalization group improved Schwarzschild black hole, and the asymptotically safe black hole will be most difficult to distinguish. Meanwhile, the leading terms of $a$ have the signs opposite to those of the leading terms of $\Omega$ and $\Gamma$, implying that the quantum effect of the quantum deformed Schwarzschild black hole will change its observables in the opposite direction from those of the renormalization group improved Schwarzschild black hole and the asymptotically safe black hole, beneficial to tell the difference among them.

Following the standard scheme [126] for the weak deflection gravitational lensing, we can find the deflection angle $\hat{\alpha}$ (9) in terms of $h=m_{\bullet} r_{0}^{-1}$ as

$$
\begin{aligned}
\hat{\alpha}(h)= & 4 h+\left(\frac{15}{4} \pi-4+\frac{3}{8} \pi a^{2}\right) h^{2} \\
& +\left[\frac{122}{3}-\frac{15}{2} \pi+\left(7-\frac{3}{4} \pi\right) a^{2}\right] h^{3} \\
& +\left[\frac{3465}{64} \pi-130+\left(\frac{825}{64} \pi-25\right) a^{2}\right. \\
& \left.+\frac{87}{256} \pi a^{4}\right] h^{4}+\mathcal{O}\left(h^{5}\right) .
\end{aligned}
$$

When the quantum deformation vanishes, i.e., $a=0, \hat{\alpha}(h)$ returns to the one of the Schwarzschild black hole [65]. The quantum deformation affects the deflection angle beginning from the second-order term of $h$, while the quantum effects of the renormalization group improved Schwarzschild black hole and the asymptotically safe black hole influence their deflection angles, respectively, starting from the third- and fourth-order terms of $h$. Since $r_{0}$ depends on the choice of the coordinates, it can be replaced with the gauge-invariant impact parameter $u$ based on Eq. (10) that

$$
\frac{u}{r_{0}}=\sqrt{\frac{r_{0}}{\sqrt{r_{0}^{2}-a_{\bullet}^{2}}-2 m_{\bullet}}} .
$$

A real-valued $u$ demands that the photon has to be outside the event horizon, i.e., $r_{0}>r_{\mathrm{H}}$. Thus, the relation between $r_{0}$ and $u$ can be solved as 


$$
\begin{aligned}
\frac{r_{0}}{u}= & 1-\frac{m_{\bullet}}{u}-\left(\frac{a^{2}}{4}+\frac{3}{2}\right) \frac{m_{\bullet}^{2}}{u^{2}}-\left(a^{2}+4\right) \frac{m_{\bullet}^{3}}{u^{3}} \\
& -\left(\frac{7}{32} a^{4}+\frac{35}{8} a^{2}+\frac{105}{8}\right) \frac{m_{\bullet}^{4}}{u^{4}}+\mathcal{O}\left(\frac{m_{\bullet}^{5}}{u^{5}}\right) .
\end{aligned}
$$

When the quantum deformation disappears, it reduces to the expression for the Schwarzschild black hole. Substituting Eq. (22) into the deflection angle $\hat{\alpha}\left(r_{0}\right)$, we obtain that

$$
\begin{aligned}
\hat{\alpha}(u)= & 4 \frac{m_{\bullet}}{u}+\left(\frac{15}{4}+\frac{3}{8} a^{2}\right) \pi \frac{m_{\bullet}^{2}}{u^{2}} \\
& +\left(\frac{128}{3}+8 a^{2}\right) \frac{m_{\bullet}^{3}}{u^{3}} \\
& +\frac{45}{64} \pi\left(77+21 a^{2}+\frac{3}{4} a^{4}\right) \frac{m_{\bullet}^{4}}{u^{4}} \\
& +\mathcal{O}\left(\frac{m_{\bullet}^{5}}{u^{5}}\right) .
\end{aligned}
$$

Likewise, the quantum deformation starts to affect the deflection angle $\hat{\alpha}(u)$ from the second-order term. When this deformation vanishes, such a deflection angle goes back to the one of the Schwarzschild black hole [65].

\subsection{Position of lensed image}

For the convenience, we define the following dimensionless variables [65]

$\beta=\frac{\mathcal{B}}{\vartheta_{\mathrm{E}}}, \quad \theta=\frac{\vartheta}{\vartheta_{\mathrm{E}}}, \quad \varepsilon=\frac{\vartheta_{\bullet}}{\vartheta_{\mathrm{E}}}$,

where $\vartheta_{\bullet}=\arctan \left(m_{\bullet} D_{\mathrm{OL}}^{-1}\right)$ is the angle subtended by black hole's gravitational radius at the observer and $\vartheta_{\mathrm{E}}$ is the angular Einstein ring radius

$\vartheta_{\mathrm{E}}=\sqrt{\frac{4 m_{\bullet} D_{\mathrm{LS}}}{D_{\mathrm{OL}} D_{\mathrm{OS}}}}$.

Among them, $\beta$ and $\theta$ are, respectively, the dimensionless angular positions of the source and the image, and $\varepsilon$ can be considered as a small parameter since we assume both the observer and the source are far away from the lens in the weak deflection gravitational lensing, making the angular gravitational radius of the black hole $\vartheta$ • much smaller than angular Einstein ring radius $\vartheta_{\mathrm{E}}$.

The smallness of $\varepsilon$ permits the Taylor expansion of the position of the lensed image $\theta$ as [65]

$\theta=\theta_{0}+\varepsilon \theta_{1}+\varepsilon^{2} \theta_{2}+\mathcal{O}\left(\varepsilon^{3}\right)$.

By making use of the deflection angle $\hat{\alpha}(u)(23)$ and the lens equation (11) by the orders of $\varepsilon$, we find the coefficients $\theta_{i}$ for the quantum deformed Schwarzschild black hole as

$$
\begin{aligned}
\theta_{0}= & \frac{1}{2}(\beta+\eta) \\
\theta_{1}= & \frac{3 \pi}{32\left(1+\theta_{0}^{2}\right)}\left(10+a^{2}\right) \\
\theta_{2}= & \frac{1}{\theta_{0}\left(1+\theta_{0}^{2}\right)^{3}}\left\{\frac{8}{3} D^{2} \theta_{0}^{8}+\left(\frac{64}{3} D^{2}-16 D\right) \theta_{0}^{6}\right. \\
& +\left(\frac{88}{3} D^{2}-32 D+16\right) \theta_{0}^{4} \\
& +\left[\frac{16}{3} D^{2}-16 D-\frac{225}{128} \pi^{2}+32\right] \theta_{0}^{2} \\
& -\frac{16}{3} D^{2}-\frac{225}{256} \pi^{2}+16 \\
& +\left[2 \theta_{0}^{4}+\left(2-\frac{45 \pi^{2}}{256}\right)\left(2 \theta_{0}^{2}+1\right)\right] a^{2} \\
& \left.-\frac{9}{1024} \pi^{2}\left(2 \theta_{0}^{2}+1\right) a^{4}\right\}
\end{aligned}
$$

where

$D=\frac{D_{\mathrm{LS}}}{D_{\mathrm{OS}}}$,

and

$\eta=\sqrt{4+\beta^{2}}$.

It is clear that $\theta_{0}$ is as the same as the one of the Schwarzschild black hole [65], not affected by any quantum deformation. The quantum deformation shows its effects from the first-order term $\theta_{1}$, so that if the astrometry can detect it, it would be possible to distinguish the quantum deformed Schwarzschild black hole from the Schwarzschild black hole. But telling its difference from the renormalization group improved Schwarzschild black hole and the asymptotically safe black hole demands extremely higher and very challenging accuracy. When the quantum deformation vanishes, i.e., $a=0, \theta_{1}$ and $\theta_{2}$ go back to those of the Schwarzschild black hole [65].

\subsection{Magnification}

The magnification $\mu$ can also be expanded in terms of $\varepsilon$ as [65]

$\mu=\mu_{0}+\varepsilon \mu_{1}+\varepsilon^{2} \mu_{2}+\mathcal{O}\left(\varepsilon^{3}\right)$. 
With the solution of $\theta$ (26), the coefficients $\mu_{i}$ can be found as

$$
\begin{aligned}
\mu_{0}= & \frac{\theta_{0}^{4}}{\theta_{0}^{4}-1}, \\
\mu_{1}= & -\frac{3 \theta_{0}^{3}}{32\left(\theta^{2}+1\right)^{3}} \pi\left(10+a^{2}\right), \\
\mu_{2}= & \frac{\theta_{0}^{2}}{\left(\theta_{0}^{2}+1\right)^{5}\left(\theta_{0}^{2}-1\right)}\left\{\frac{8}{3} D^{2} \theta_{0}^{8}\right. \\
& +4\left(12 D^{2}-8 D-8\right) \theta_{0}^{6} \\
& +\left(\frac{272}{3} D^{2}-64 D+\frac{675}{128} \pi^{2}-64\right) \theta_{0}^{4} \\
& +16\left(3 D^{2}-2 D-2\right) \theta_{0}^{2}+\frac{8}{3} D^{2} \\
& -\left[4 \theta_{0}^{6}-\left(\frac{135}{128} \pi^{2}+8\right) \theta_{0}^{4}-4 \theta_{0}^{2}\right] a^{2} \\
& \left.+\frac{27}{512} \pi^{2} \theta_{0}^{4} a^{4}\right\} .
\end{aligned}
$$

Similarly, the leading term of the magnification $\mu_{0}$ is not affected by the quantum deformation, which starts to appear in the first-order term $\mu_{1}$. When the quantum deformation vanishes, i.e., $a=0, \mu_{1}$ and $\mu_{2}$ reduce to their corresponding values for the Schwarzschild black hole [65].

\subsection{Time delay}

The time function $T(R)$ (16) can be expanded in terms of $h=m_{\bullet} r_{0}^{-1}$ as

$T(R)=T_{0}+r_{0} T_{1} h+r_{0} T_{2} h^{2}+\mathcal{O}\left(h^{3}\right)$,

where

$$
\begin{aligned}
T_{0}= & \sqrt{R^{2}-r_{0}^{2}}, \\
T_{1}= & \frac{\sqrt{1-\xi^{2}}}{1+\xi}+2 \log \left(\frac{1+\sqrt{1-\xi^{2}}}{\xi}\right), \\
T_{2}= & -\frac{(4+5 \xi) \sqrt{1-\xi^{2}}}{2(1+\xi)^{2}} \\
& +\frac{3}{8}(\pi-2 \arcsin \xi)\left(10+a^{2}\right),
\end{aligned}
$$

with

$\xi=\frac{r_{0}}{R}$.

The quantum deformation only affects the second-order term $T_{2}$, leaving the geometric term $T_{0}$ and the Shapiro delay term $T_{1}$ unchanged as the same as those of the Schwarzschild black hole [65]. If $a=0, T_{2}$ returns to the value for the Schwarzschild black hole [65].

The time delay $\tau$ is defined as the difference between the travel time of a photon from the source to the observer with

and without the gravitational lensing that reads [65]

$c \tau=T\left(R_{\mathrm{src}}\right)+T\left(R_{\mathrm{obs}}\right)-\frac{D_{\mathrm{OS}}}{\cos \mathcal{B}}$.

Rescaled by the time $\tau_{\mathrm{E}}$ which is defined as

$\tau_{\mathrm{E}}=\frac{4 m_{\bullet}}{c}$,

the dimensionless time delay can be found in terms of $\varepsilon$ as

$$
\begin{aligned}
\hat{\tau}= & \frac{\tau}{\tau_{\mathrm{E}}} \\
= & \frac{1}{2}\left[1+\beta^{2}-\theta_{0}^{2}-\log \left(\frac{D_{\mathrm{OL}}}{4 D_{\mathrm{LS}}} \theta_{0}^{2} \vartheta_{E}^{2}\right)\right] \\
& +\frac{3 \pi}{32 \theta_{0}} \varepsilon\left(10+a^{2}\right)+\mathcal{O}\left(\varepsilon^{2}\right),
\end{aligned}
$$

where the quantum deformation begins to play a role from the first-order term of $\varepsilon$. When $a=0$, the time delay $\hat{\tau}$ has the same form as the one of the Schwarzschild black hole. Since the quantum deformed Schwarzschild black hole and the Schwarzschild black hole share the same leading term of the time delay, distinguishing them by measuring the time delay requires the accuracy at least up to the first-order term of $\varepsilon$.

\subsection{Relations among positions and magnification}

In this work, we adopt the convention in Ref. [65] that the angular position of the lensed image is set to be positive. When the image is on the same side of the source with respect to the lens, it is called the positive-parity image with the position angle $\beta>0$ denoted by the symbol of " + "; otherwise, the image on the other side is the negative-parity one with $\beta<0$ denoted by the symbol of "-".

\subsubsection{Relations of images positions}

Taking $\beta>0$ and $\beta<0$, we can find the positions of positive- and negative-parity images $\theta^{ \pm}$as

$$
\begin{aligned}
\theta_{0}^{ \pm}= & \frac{1}{2}(\eta \pm|\beta|) \\
\theta_{1}^{ \pm}= & \frac{3 \pi}{16(\eta \pm|\beta|) \eta}\left(10+a^{2}\right) \\
\theta_{2}^{ \pm}= & \frac{1}{\eta^{3}(\eta \pm|\beta|)^{4}}\left\{\left[\frac{64}{3} D^{2} \beta^{8}+\frac{128}{3} D(8 D-3) \beta^{6}\right.\right. \\
& +\frac{64}{3}\left(79 D^{2}-48 D+6\right) \beta^{4} \\
& +\left(\frac{8576}{3} D^{2}-2304 D-\frac{225}{16} \pi^{2}+768\right) \beta^{2} \\
& \left.+\frac{2560}{3} D^{2}-1024 D-\frac{675}{16} \pi^{2}+1024\right] \\
& +\left[16 \beta^{4}+\left(96-\frac{45}{16} \pi^{2}\right) \beta^{2}+128-\frac{135}{16} \pi^{2}\right] a^{2}
\end{aligned}
$$




$$
\begin{aligned}
& \left.-\frac{9}{64}\left(\beta^{2}+3\right) \pi^{2} a^{4}\right\} \\
& \pm \frac{\eta|\beta|}{\eta^{3}(\eta \pm|\beta|)^{4}}\left\{\left[\frac{64}{3} D^{2} \beta^{6}+\frac{128}{3}(7 D-3) D \beta^{4}\right.\right. \\
& +\frac{64}{3}\left(53 D^{2}-36 D+6\right) \beta^{2} \\
& \left.+\frac{3328}{3} D^{2}-1024 D-\frac{225}{16} \pi^{2}+512\right] \\
& \left.+\left(16 \beta^{2}+64-\frac{45}{16} \pi^{2}\right) a^{2}-\frac{9}{64} \pi^{2} a^{4}\right\}
\end{aligned}
$$

which lead to the following relations

$$
\begin{aligned}
\theta_{0}^{+} \theta_{0}^{-}=1 & \\
\theta_{0}^{+}-\theta_{0}^{-} & =|\beta| \\
\theta_{1}^{+}+\theta_{1}^{-} & =\frac{3}{32} \pi\left(10+a^{2}\right) \\
\theta_{1}^{+}-\theta_{1}^{-} & =-\frac{3}{32 \eta} \pi|\beta|\left(10+a^{2}\right) \\
\theta_{2}^{+}-\theta_{2}^{-} & =|\beta|\left[8 D^{2}-16+\frac{225}{256} \pi^{2}\right. \\
& \left.+\left(\frac{45}{256} \pi^{2}-2\right) a^{2}+\frac{9}{1024} \pi^{2} a^{4}\right] .
\end{aligned}
$$

The zero-order term $\theta_{0}^{ \pm}$and the relations between them are not affected by the quantum deformation, which starts to play a role from the next-to-leading-order terms. The first- and second-order terms $\theta_{1}^{ \pm}$and $\theta_{2}^{ \pm}$and their relations $\theta_{1}^{+}+\theta_{1}^{-}$ and $\theta_{1}^{+}-\theta_{1}^{-}$are all influenced by the quantum deformation. When such a deformation disappears, i.e., $a=0$, these terms and their relations reduce to those of the Schwarzschild black hole [65].

\subsubsection{Relations of magnification}

According to the magnification (32), its values for the positive- and negative-parity images $\mu^{ \pm}$can be found as

$$
\begin{aligned}
\mu_{0}^{ \pm}= & \frac{1}{2} \pm \frac{\beta^{2}+2}{2|\beta| \eta} \\
\mu_{1}^{+}= & \mu_{1}^{-}=-\frac{3}{32} \frac{\pi}{\eta^{3}}\left(10+a^{2}\right), \\
\mu_{2}^{ \pm}= & \pm \frac{1}{\eta^{5}|\beta|}\left[\frac{8}{3} D^{2} \beta^{4}+\left(\frac{176}{3} D^{2}-32 D-32\right) \beta^{2}\right. \\
& +192 D^{2}-128 D+\frac{675}{128} \pi^{2}-128 \\
& \left.-\left(4 \beta^{2}-\frac{135}{128} \pi^{2}+16\right) a^{2}+\frac{27}{512} \pi^{2} a^{4}\right],
\end{aligned}
$$

which have the relations

$\mu_{0}^{+}+\mu_{0}^{-}=1$,

$\mu_{0}^{+}-\mu_{0}^{-}=\frac{\beta^{2}+2}{|\beta| \eta}$

$\mu_{1}^{+}-\mu_{1}^{-}=0$,

$\mu_{2}^{+}+\mu_{2}^{-}=0$.

The quantum deformation affects the first- and secondorder terms $\mu_{1}^{ \pm}$and $\mu_{2}^{ \pm}$but leaves the zero-order term $\mu_{0}^{ \pm}$ unchanged. Even though, the combinations of them listed above are immune to any quantum deformation. When $a=0$, these magnification and their relations all go back to those of the Schwarzschild black hole [65].

\subsubsection{Total magnification and centroid}

In the weak deflection gravitational lensing, if the positiveand negative-parity images can not be resolved, then the total magnification and the centroid of these two images will be the observables. The total magnification is defined as

$$
\begin{aligned}
\mu_{\mathrm{tot}} & =\left|\mu^{+}\right|+\left|\mu^{-}\right|=\mu^{+}-\mu^{-} \\
& =\frac{\beta^{2}+2}{|\beta| \eta}+2 \varepsilon^{2} \mu_{2}^{+}+\mathcal{O}\left(\varepsilon^{3}\right) .
\end{aligned}
$$

The leading term of $\mu_{\text {tot }}$ depends only on the angular position of the source, while the quantum deformation begins to affect the total magnification from the second order of $\varepsilon$. For its firstorder term, Eq. (57) ensures that it will never appear in the total magnification. Hence, any difference in the total magnification between the quantum deformed Schwarzschild black hole and Schwarzschild black hole has to be told by measuring its second-order term, which is very challenging for current ability of photometry. When the quantum deformation vanishes, i.e., $a=0$, the total magnification reduces to the value for Schwarzschild black hole [65].

The centroid $\Theta_{\text {cent }}$ is defined as the weighted average of the magnification by the two lensed images' positions, and it reads as

$\Theta_{\text {cent }}=\frac{\theta^{+}\left|\mu^{+}\right|-\theta^{-}\left|\mu^{-}\right|}{\left|\mu^{+}\right|+\left|\mu^{-}\right|}=\frac{\theta^{+} \mu^{+}+\theta^{-} \mu^{-}}{\mu^{+}-\mu^{-}}$,

which can be found in terms of $\varepsilon$ as

$$
\begin{aligned}
\Theta_{\text {cent }}= & \frac{|\beta|\left(\beta^{2}+3\right)}{\beta^{2}+2}+\frac{|\beta| \varepsilon^{2}}{\left(\beta^{2}+2\right)^{2} \eta^{2}}\left[\frac{8}{3} D^{2} \beta^{6}\right. \\
& +\left(\frac{104}{3} D^{2}-16 D\right) \beta^{4} \\
& +\left(\frac{272}{3} D^{2}-64 D+32\right) \beta^{2} \\
& -\frac{64}{3} D^{2}+128-\frac{675}{128} \pi^{2}
\end{aligned}
$$




$$
\begin{aligned}
& +\left(4 \beta^{2}+16-\frac{135}{128} \pi^{2}\right) a^{2} \\
& \left.-\frac{27}{512} \pi^{2} a^{4}\right]+\mathcal{O}\left(\varepsilon^{3}\right) .
\end{aligned}
$$

Similar to the total magnification, the leading term of the centroid $\Theta_{\text {cent }}$ is not affected by the quantum deformation and its first-order term cancels out. The quantum deformation only has its influence on the second-order term of the centroid. When it vanishes, $\Theta_{\text {cent }}$ returns to the one of the Schwarzschild black hole [65].

\subsubsection{Differential time delay}

The time delay of one lensed image is not observable since the travel time of an unlensed photon is unknown. Instead, the differential time delay between the positive- and negativeparity images can be measured in practice. The definition of differential time delay $\Delta \hat{\tau}$ is

$$
\begin{aligned}
\Delta \hat{\tau}= & \hat{\tau}^{+}-\hat{\tau}^{-} \\
= & \left(\frac{1}{2}|\beta| \eta+\log \frac{\eta+|\beta|}{\eta-|\beta|}\right) \\
& +\frac{3}{32} \pi \varepsilon\left(10+a^{2}\right)|\beta|+\mathcal{O}\left(\varepsilon^{2}\right) .
\end{aligned}
$$

While its leading term is immune to the quantum deformation, its first-order them is affected. When $a=0$, the differential time delay $\Delta \hat{\tau}$ reduces to the one of the Schwarzschild black hole [65].

As shown in the aforementioned results, the quantum deformation affects the first-order terms of the image position, the magnification and the differential time delay of the lensed images, while it only changes the second-order terms of the total magnification and the centroid. Therefore, it is easier to distinguish the quantum deformed Schwarzschild black hole from the Schwarzschild black hole by detecting the deviations of the image position, the magnification and the differential time delay. As a comparison, the quantum effect of the renormalization group improved Schwarzschild black hole merely has its influence on the second-order terms of the image position, the magnification and the differential time delay in its weak deflection gravitational lensing [113], while the asymptotically safe black hole will have completely the same observables as those of the Schwarzschild black hole. It suggests that, based on measuring the observables of the weak deflection gravitational lensing, the quantum deformed Schwarzschild black hole would be much more easily told difference from the Schwarzschild black hole than the renormalization group improved Schwarzschild black hole, whereas the asymptotically safe black hole is indistinguishable from the Schwarzschild black hole.

\subsection{Practical observables}

In the realistic astronomical observations, the practical observables of the weak deflection gravitational lensing include the angular separation $P_{\text {tot }}$, the difference of the angular positions $\Delta P$, the total flux $F_{\text {tot }}$, the difference of the flux $\Delta F$, the centroid $S_{\text {cent }}$ and the differential time delay $\Delta \tau$ of the lensed images [66]. These practical observables can be obtained from the scaled quantities $(\beta, \theta, \mu, \hat{\tau})$, where the flux of a lensed image is the magnified one of the source, i.e., $F=|\mu| F_{\text {src }}$. Keeping the leading contributions of the quantum deformation, we find the practical observables of the weak deflection gravitational lensing by the quantum deformed Schwarzschild black hole as

$$
\begin{aligned}
P_{\mathrm{tot}} & =\vartheta^{+}+\vartheta^{-} \\
& =\mathcal{E}+\frac{3}{32} \varepsilon \vartheta_{\mathrm{E}} \pi\left(10+a^{2}\right)+\mathcal{O}\left(\varepsilon^{2}\right), \\
\Delta P & =\vartheta^{+}-\vartheta^{-} \\
& =|\mathcal{B}|\left[1-\frac{3}{32} \varepsilon \pi \frac{\vartheta_{\mathrm{E}}}{\mathcal{E}}\left(10+a^{2}\right)+\mathcal{O}\left(\varepsilon^{2}\right)\right], \\
F_{\mathrm{tot}} & =F^{+}+F^{-} \\
= & \frac{F_{\mathrm{src}}}{|\mathcal{B}| \mathcal{E}}\left(\mathcal{B}^{2}+2 \vartheta_{\mathrm{E}}^{2}\right)+\mathcal{O}\left(\varepsilon^{2}\right), \\
\Delta F= & F^{+}-F^{-} \\
= & F_{\mathrm{src}}\left[1-\frac{3}{16} \varepsilon \pi \frac{\vartheta_{E}^{3}}{\mathcal{E}^{3}}\left(10+a^{2}\right)\right]+\mathcal{O}\left(\varepsilon^{2}\right), \\
S_{\mathrm{cent}}= & \frac{\vartheta^{+} F^{+}-\vartheta^{-} F^{-}}{F^{+}+F^{-}} \\
= & \frac{|\mathcal{B}|}{\mathcal{B}^{2}+2 \vartheta_{E}^{2}}\left(\mathcal{B}^{2}+3 \vartheta_{E}^{2}\right)+\mathcal{O}\left(\varepsilon^{2}\right), \\
\Delta \tau= & \frac{D_{\mathrm{OL}} D_{\mathrm{OS}}}{c D_{\mathrm{LS}}}\left\{\frac{1}{2}|\mathcal{B}| \mathcal{E}+\vartheta_{E}^{2} \log \left(\frac{\mathcal{E}+|\mathcal{B}|}{\mathcal{E}-|\mathcal{B}|}\right)\right. \\
& \left.+\frac{3}{32} \varepsilon \pi|\mathcal{B}| \vartheta_{E}\left(10+a^{2}\right)+\mathcal{O}\left(\varepsilon^{2}\right)\right\}, \\
\Delta &
\end{aligned}
$$

where

$\mathcal{E}=\sqrt{\mathcal{B}^{2}+4 \vartheta_{E}^{2}}$

In order to indicate the effects of the quantum deformation on these observables of the weak deflection gravitational lensing, we define their deviations from those of the Schwarzschild black hole as

$$
\begin{aligned}
& \delta P_{\text {tot }}=P_{\text {tot }}-\left.P_{\text {tot }}\right|_{a=0}=\frac{3 \pi}{32} \varepsilon \vartheta_{\mathrm{E}} a^{2}+\mathcal{O}\left(\varepsilon^{2}\right) \\
& \delta \Delta P=\Delta P-\left.\Delta P\right|_{a=0}=-\frac{3 \pi}{32 \mathcal{E}} \varepsilon|\mathcal{B}| \vartheta_{\mathrm{E}} a^{2}+\mathcal{O}\left(\varepsilon^{2}\right) \\
& \delta r_{\text {tot }}=2.5 \log _{10}\left(\frac{F_{\text {tot }}}{\left.F_{\text {tot }}\right|_{a=0}}\right)=\mathcal{O}\left(\varepsilon^{2}\right)
\end{aligned}
$$




$$
\begin{aligned}
\delta \Delta r & =2.5 \log _{10}\left(\frac{\Delta F}{\left.\Delta F\right|_{a=0}}\right) \\
& =-\frac{15 \pi}{32 \log (10)} \varepsilon \frac{\vartheta_{\mathrm{E}}^{3}}{\mathcal{E}^{3}} a^{2}+\mathcal{O}\left(\varepsilon^{2}\right), \\
\delta S_{\text {cent }} & =S_{\text {cent }}-\left.S_{\text {cent }}\right|_{a=0}=\mathcal{O}\left(\varepsilon^{2}\right), \\
\delta \Delta \tau & =\Delta \tau-\left.\Delta \tau\right|_{a=0} \\
& =\frac{D_{\mathrm{OL}} D_{\mathrm{OS}}}{c D_{\mathrm{LS}}} \frac{3 \pi}{32} \varepsilon|\mathcal{B}| \vartheta_{\mathrm{E}} a^{2}+\mathcal{O}\left(\varepsilon^{2}\right),
\end{aligned}
$$

where the quantities about the fluxes are converted into the magnitude of brightness by the astronomical convention. $P_{\mathrm{tot}}, \Delta P, \Delta F$ and $\Delta \tau$ are at the order of $\mathcal{O}(\varepsilon)$, while $\delta r_{\text {tot }}$ and $S_{\text {cent }}$ are at the order of $\mathcal{O}\left(\varepsilon^{2}\right)$, meaning that these two deviations are much harder to detect and far beyond the reach of current capabilities. For comparison, the deviations of the observables in the weak deflection gravitational lensing by the renormalization group improved Schwarzschild black hole are at the order of $\mathcal{O}\left(\varepsilon^{2}\right)$ or higher [113], whereas these deviations for the asymptotically safe black hole are even smaller. It suggests that the quantum deformed Schwarzschild black hole would be most easily distinguished than the renormalization group improved Schwarzschild black hole and the asymptotically safe black hole.

\subsection{Example of Sgr A*}

We take the supermassive black hole, Sgr A*, as the lens with the mass $m_{\bullet, \mathrm{SgrA} *}=4.28 \times 10^{6} \mathrm{M}_{\odot}$ and the distance $D_{\mathrm{OL}, \mathrm{SgrA} *}=8.32 \mathrm{kpc}[128]$ and calculate the values of these observables for $10^{-2}<\beta<10$ and on the domain $\mathcal{D}$ (8) which is determined based on the observed shadow of M87* (see Sect. 4.1 for details). The left panel of Fig. 2 shows the angular separation $P_{\text {tot }}$, the position difference $\Delta P$, the flux difference $\Delta F$ and the differential time delay $\Delta \tau$ between the two lensed images in the weak deflection gravitational lensing by the quantum deformed Schwarzschild black hole for Sgr A*. Its right panel demonstrates the deviations of these observables $\delta P_{\text {tot }}, \delta \Delta P, \delta \Delta r$ and $\delta \Delta \tau$ from those of the Schwarzschild black hole.

The angular separation between the two lensed images $P_{\text {tot }}$ ranges from about 1.4 to 7.3 milliarcsecond (mas). It increases with the angular position of the source $\beta$, but it is not sensitive to the change of the quantum deformation. With currently angular resolution about 3 mas for GRAVITY [129], it would be possible to resolve the positive- and negative-parity images in the weak deflection gravitational lensing if $P_{\text {tot }}$ could reach about 7 mas for a large $\beta$. That ensures the feasibility to detect other observables. Its deviation from the one of the Schwarzschild black hole $\delta P_{\text {tot }}$ changes from 0 to 3.5 microarcsecond ( $\mu$ as). In contrast to $P_{\text {tot }}, \delta P_{\text {tot }}$ significantly depends on the quantum defor- mation and is hardly affected by $\beta . \delta P_{\text {tot }}$ is below the currently astrometric accuracy of GRAVITY about 10-20 $\mu$ as [129] and, therefore, it is not presently possible to distinguish the quantum deformed Schwarzschild black hole from the Schwarzschild black hole by measuring such an observable. The difference of the angular positions of the two lensed images $\Delta P$ varies from about 0.01 to 7.0 mas. It grows as $\beta$, but is barely changed by the quantum deformation. It would also be possible to resolve $\Delta P$ by GRAVITY at best conditions. Its deviation from the one of the Schwarzschild black hole $\delta \Delta P$ ranges from -3.4 to $0 \mu$ as, below the astrometric accuracy of GRAVITY. However, with further improvement [130], the upgraded GRAVITY + might have sufficient ability to detect the small changes of $\delta P_{\text {tot }}$ and $\delta \Delta P$ in the near future. The normalized fluxes difference between the lensed images $\Delta F / F_{\text {src }}$ is very close to 1 for a large $\beta$ and decrease to about 0.993 for a small $\beta$. It is almost immune to the quantum deformation. This suggests that the fluxes of positive- and negative-parity images are quite different when $\beta \sim 10$, indicating the faintness of the negative-parity image. Its deviation from the one of the Schwarzschild black hole $\delta \Delta r$ changes from about $-1.3 \times 10^{-3}$ to 0 mag. When $\beta \lesssim 1$, $\delta \Delta r$ is significantly affected by the quantum deformation and is well within the current ability of space-borne photometry [131] as long as the flares of Sgr A* are quiescent. The differential time delay between the lensed images $\Delta \tau$ ranges from a few tens of seconds to about $1.3 \mathrm{~h}$. It grows with respect to $\beta$ and is barely changed by the quantum deformation. Its deviation from the one of the Schwarzschild black hole $\delta \Delta \tau$ can reach its peak of about $4 \mathrm{~s}$ when $\beta \sim 10$ and the quantum deformation is $a \sim 1.5$. Since a typical dataset of $\mathrm{Sgr} \mathrm{A}^{*}$ and S2 observed by GRAVITY contains 30 exposures with an individual integration time of $10 \mathrm{~s}[132,133], \Delta \tau$ might be able to measure whereas $\delta \Delta \tau$ is mildly shorter than the individual integration time and, thus, undetectable.

In summary, we find that (1) the angular separation $P_{\text {tot }}$, the angular difference $\Delta P$, the fluxes difference $\Delta F$ and the time delay $\Delta \tau$ are possible to detect with current technology; (2) the quantum deformed Schwarzschild black hole might be distinguished from the Schwarzschild black hole by measuring the $\delta \Delta r$ during a quiet state of $\mathrm{Sgr} \mathrm{A}^{*}$ with a dedicated space telescope; and (3) it would also be feasible by detecting $\delta P_{\text {tot }}, \delta \Delta P$ and $\delta \Delta \tau$ with GRAVITY+ in the near future.

For comparison, the absolute deviations of these observables for the renormalization group improved Schwarzschild black hole are smaller than those of the quantum deformed Schwarzschild black hole by about 2-3 orders of magnitude: $\left|\delta P_{\mathrm{tot}, \mathrm{RG}}\right|<53$ nanoarcsecond (nas), $\left|\delta \Delta P_{\mathrm{RG}}\right|<24$ nas, $\left|\delta r_{\text {tot, RG }}\right|<31 \mu \mathrm{mag},\left|\delta \Delta r_{\mathrm{RG}}\right| \sim \mathcal{O}\left(\varepsilon^{3}\right),\left|\delta S_{\text {cent }, \mathrm{RG}}\right|<$ 10 nas and $\left|\delta \Delta \tau_{\mathrm{RG}}\right|<3 \mathrm{~ms}$ [113]. The absolute deviations for the asymptotically safe black hole are even smaller by about $4-5$ orders of magnitude: $\left|\delta P_{\text {tot, AS }}\right|<0.6$ nas, $\left|\delta \Delta P_{\mathrm{AS}}\right|<0.4$ nas, $\left|\delta r_{\text {tot, } \mathrm{AS}}\right|<0.6 \mu \mathrm{mag},\left|\delta \Delta r_{\mathrm{AS}}\right|<$ 

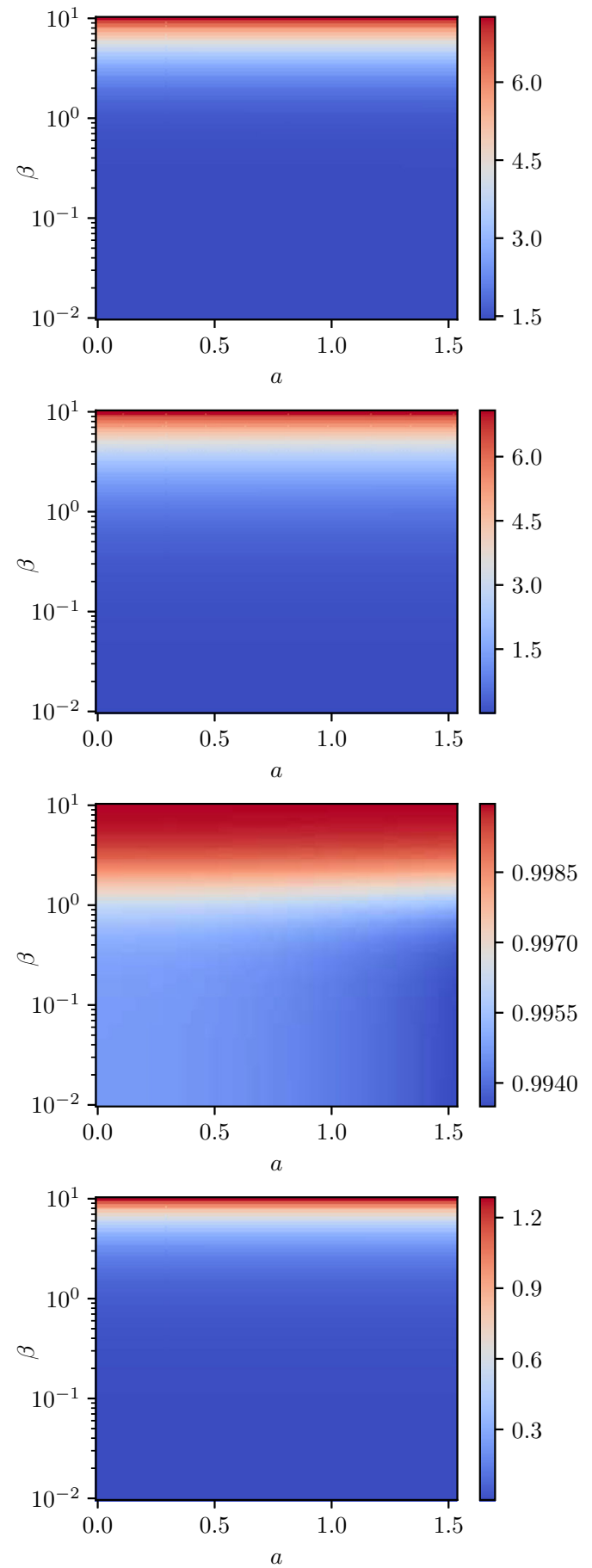

Fig. 2 Color-indexed practical observables in the weak deflection gravitational lensing by the quantum deformed Schwarzschild black hole and their deviations from those of the Schwarzschild black hole are showed with respect to the source position $\beta$ and the quantum defor-

$0.5 \mu \mathrm{mag},\left|\delta S_{\text {cent, } \mathrm{AS}}\right|<0.3$ nas and $\left|\delta \Delta \tau_{\mathrm{AS}}\right| \sim \mathcal{O}\left(\varepsilon^{4}\right)$. Therefore, among these quantum black holes, the quantum deformed Schwarzschild black hole might be most easily distinguished from the Schwarzschild black hole through the
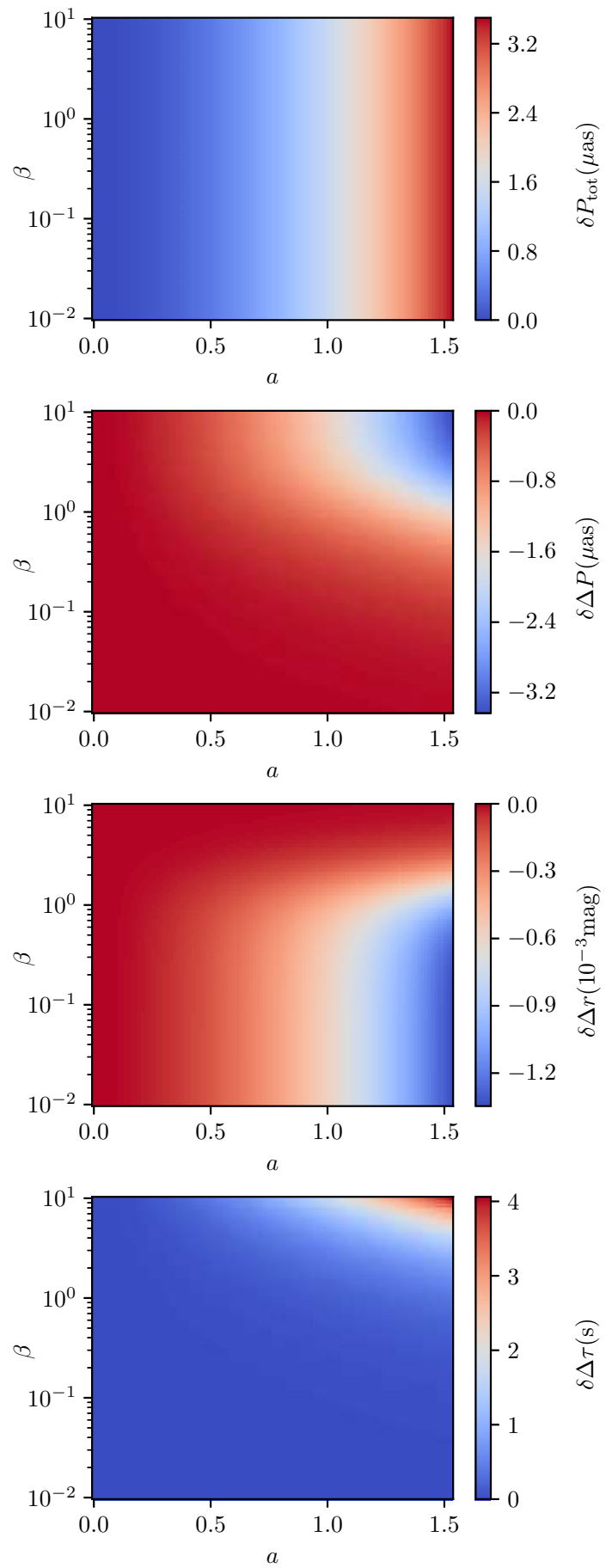

mation $a$ for Sgr A*. From top to bottom, the left panel demonstrates $P_{\text {tot }}, \Delta P, \Delta F$ and $\Delta \tau$; and the right panel displays $\delta P_{\text {tot }}, \delta \Delta P, \delta \Delta r$ and $\delta \Delta \tau$

weak deflection gravitational lensing, while the renormalization group improved Schwarzschild black hole and asymptotically safe black hole would be much harder to tell their difference in the future. 


\section{Strong deflection gravitational lensing}

In the strong deflection gravitational lensing, the light ray passes the vicinity of the quantum deformed Schwarzschild black hole. The closest distance between the photon and the black hole $r_{0}$ is comparable with its gravitational radius. When a photon approaches the photon sphere of the black hole, its deflection angle diverges. Therefore, it can loop around the black hole before reaching the observer, producing infinite number of relativistic images, which is a unique feature not present in the weak deflection gravitational lensing.

\subsection{Photon sphere and shadow}

The photon sphere is the unstable circular orbit of a photon. Its radius $r_{\mathrm{m}}$ is defined as the largest non-negative root of the following equation $[72,134]$

$\frac{A^{\prime}(r)}{A(r)}=\frac{C^{\prime}(r)}{C(r)}$.

where' denotes the differentiation against $r$. The quantum deformed Schwarzschild black hole has the photon sphere with the radius as

$r_{\mathrm{m}}=\sqrt{\frac{3}{2}} \sqrt{a_{\bullet}^{2}+3 m_{\bullet}^{2}+m_{\bullet} \sqrt{2 a_{\bullet}^{2}+9 m_{\bullet}^{2}}}$.

When the quantum deformation vanishes, i.e., $a_{\bullet}=0$, it returns to the one of the Schwarzschild black hole $r_{\mathrm{m}, \mathrm{Sch}}=$ $3 m$. For convenience, we define the dimensionless quantity

$x_{\mathrm{m}}=\frac{r_{\mathrm{m}}}{m_{\bullet}}=\sqrt{\frac{3}{2}} \sqrt{a^{2}+3+\sqrt{2 a^{2}+9}}$,

where $a$ is the dimensionless quantum deformation and defined in Eq. (6). Figure 3a shows $x_{\mathrm{H}}$ and $x_{\mathrm{m}}$ with respect to the quantum deformation $a$. This photon sphere would cast a shadow. The radius of the shadow of the quantum deformed Schwarzschild black hole is

$r_{\mathrm{sh}}=\frac{r_{\mathrm{m}}}{\sqrt{A_{\mathrm{m}}}}$,

where and hereafter a subscript " $m$ " stands for the evaluation of a quantity at $r=r_{\mathrm{m}}$. We also define a dimensionless quantity $x_{\mathrm{sh}}=r_{\mathrm{sh}} m_{\bullet}^{-1}$. Figure $3 \mathrm{~b}, \mathrm{c}$ show $x_{\mathrm{sh}}$ and the ratio of $x_{\mathrm{Sh}}$ to the event horizon radius $x_{\mathrm{H}}$, respectively. It is clear that both $x_{\mathrm{m}}$ and $x_{\mathrm{sh}}$ increase with the growth of $a$, while the ratio decreases as $a$ that agrees with the statement of Ref. [58].

The direct image of the shadow of M87* by EHT [2], which is consistent with the prediction by GR within $17 \%$ at the 68-percentile level, provides a way to probe the strong gravitational field region of a black hole and constrain the theories of gravitation [125]. Following the approach of Ref.

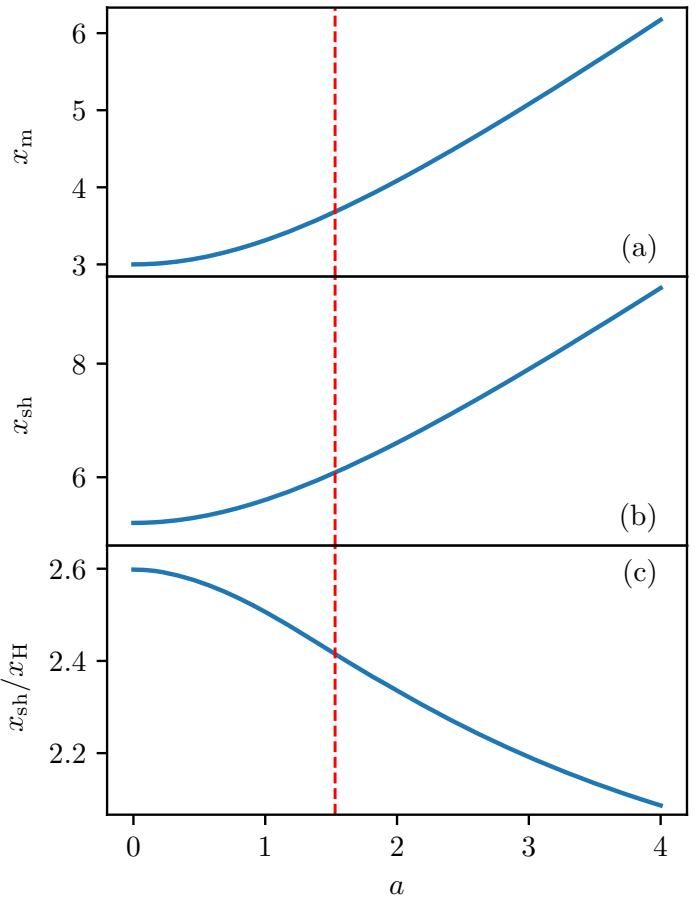

Fig. 3 From top to bottom, it shows the (dimensionless) radii of the photon sphere $x_{\mathrm{m}}$, the shadow $x_{\mathrm{sh}}$ and their ratio for the quantum deformed Schwarzschild black hole with respect to the quantum deformation $a$. The red dashed line marks the upper bound on $a$ given by the EHT observation

[125] and making use of Eq. (79), we can obtain the preliminary bound on the quantum deformation based on the observed size of M87*'s shadow as (see the red dashed line in Fig. 3)

$\mathcal{D}=\{a \mid 0<a<1.53\}$.

It is worth mentioning that although the spin of the black hole and its inclination are not taken into account, their combined effects on the size of the shadow and the circularity of its shape are no more than 4 and 7\%, respectively [135]. Therefore, we expect that such a bound on the dimensionless quantum deformation $a$ is valid at least for the leading order and the EHT observation can be used to test the nonrotating quantum deformed Schwarzschild black hole sufficiently. For the exact quantum deformation $a_{\bullet}=a m_{\bullet}$, it depends on the mass of the black hole so that $a_{\bullet}$ might have very different values for various black holes even though all of them have the same $a$.

\subsection{Observables}

With the method of the strong deflection limit [73], the deflection angle in the strong deflection gravitational lensing can be found as 


$$
\begin{aligned}
\hat{\alpha}(\vartheta)= & -\bar{a} \log \left(\frac{\vartheta D_{\mathrm{OL}}}{u_{\mathrm{m}}}-1\right)+\bar{b} \\
& +\mathcal{O}\left[\left(u-u_{\mathrm{m}}\right) \log \left(u-u_{\mathrm{m}}\right)\right],
\end{aligned}
$$

with

$$
\begin{aligned}
& \bar{a}= \sqrt{\frac{2}{A_{\mathrm{m}} C_{\mathrm{m}}^{\prime \prime}-A_{\mathrm{m}}^{\prime \prime} C_{\mathrm{m}}}}, \\
& \bar{b}=-\pi+\bar{a} \log \left[\frac{\left(1-A_{\mathrm{m}}\right)^{2}}{A_{\mathrm{m}}^{\prime 2}}\left(\frac{C_{\mathrm{m}}^{\prime \prime}}{C_{\mathrm{m}}}-\frac{A_{\mathrm{m}}^{\prime \prime}}{A_{\mathrm{m}}}\right)\right] \\
&+2 \int_{0}^{1}\left[\frac{1-A_{\mathrm{m}}}{A_{z}^{\prime} C_{z} \sqrt{A_{\mathrm{m}} C_{\mathrm{m}}^{-1}-A_{z} C_{z}^{-1}}}-\frac{\bar{a}}{z}\right] \mathrm{d} z
\end{aligned}
$$

and

$z=\frac{A(r)-A_{\mathrm{m}}}{1-A_{\mathrm{m}}}$

where" denotes the second derivative against $r$ and a quantity with subscript $z$ means its dependence on $z$.

If it is assumed that the observer and source are far away from the lens and that the source, lens and observer are nearly aligned, the lens equation in the scenario of the strong deflection gravitational lensing can be simplified as [136]

$\mathcal{B}=\vartheta-\frac{D_{\mathrm{LS}}}{D_{\mathrm{OS}}} \Delta \hat{\alpha}$

where $\Delta \hat{\alpha}=\hat{\alpha}-2 n \pi$ and $n$ is the number of loops that the photon takes around the black hole. Combining the logarithmic divergence of the deflection angle (80) and the lens equation (84), we can find that the angular separation between the relativistic images decreases exponentially with $n$, making them difficult to be resolved.

In the strong deflection gravitational lensing, the travel time taken by a photon from the source to the observer can be obtained by Eq. (16). With the assumption that the closest approaches between two photons and the lens are, respectively, $r_{0,1}$ and $r_{0,2}$, the differential time delay between these two relativistic images can be found as [137]

$T_{1}-T_{2}=\tilde{T}\left(r_{0,1}\right)-\tilde{T}\left(r_{0,2}\right)+2 \int_{r_{0,1}}^{r_{0,2}} \frac{1}{A(r)} \mathrm{d} r$.

Making use of the same method for dealing with the deflection angle (80), the time function $\tilde{T}(r)$ can also be obtained as [137]

$$
\begin{aligned}
\tilde{T}= & -\tilde{a} \log \left(\frac{u}{u_{\mathrm{m}}}-1\right)+\tilde{b} \\
& +\mathcal{O}\left[\left(u-u_{\mathrm{m}}\right) \log \left(u-u_{\mathrm{m}}\right)\right],
\end{aligned}
$$

where

$$
\begin{aligned}
\tilde{a}= & \bar{a} u_{\mathrm{m}}, \\
\tilde{b}= & -\pi+\tilde{a} \log \left[\frac{\left(1-A_{\mathrm{m}}\right)^{2}}{A_{\mathrm{m}}^{\prime 2}}\left(\frac{C_{\mathrm{m}}^{\prime \prime}}{C_{\mathrm{m}}}-\frac{A_{\mathrm{m}}^{\prime \prime}}{A_{\mathrm{m}}}\right)\right] \\
& +2 \int_{0}^{1}\left[\frac{1-A_{\mathrm{m}}}{A_{z}^{\prime}} \frac{\sqrt{A_{\mathrm{m}}}}{A_{z}}\left(\sqrt{\frac{A_{\mathrm{m}} C_{z}}{A_{\mathrm{m}} C_{z}-A_{z} C_{\mathrm{m}}}}-1\right)\right. \\
& \left.-\frac{\tilde{a}}{z}\right] \mathrm{d} z .
\end{aligned}
$$

When the first relativistic image $(n=1)$ can only be resolved and other images $(n \geq 2)$ are packed together, the observables in the strong deflection gravitational lensing are the angular radius of the photon sphere (shadow) $\theta_{\infty}$ as well as the angular separation $s$ and magnitude difference $\Delta r$ between the first relativistic image and the packed others [73]

$$
\begin{aligned}
\theta_{\infty} & =\frac{u_{\mathrm{m}}}{D_{\mathrm{OL}}}, \\
s & =\theta_{\infty} \exp \left(\frac{\bar{b}}{\bar{a}}-\frac{2 \pi}{\bar{a}}\right), \\
\Delta r & =\frac{5 \pi}{\bar{a} \log 10} .
\end{aligned}
$$

If the first and second relativistic images can be separated, their differential time delay can be found as [137]

$\Delta T_{2,1}=\Delta T_{2,1}^{0}+\Delta T_{2,1}^{1}$,

where the perimeter term $\Delta T_{2,1}^{0}$ and the exponential term $\Delta T_{2,1}^{1}$ are

$$
\begin{aligned}
\Delta T_{2,1}^{0}= & 2 \pi u_{\mathrm{m}}, \\
\Delta T_{2,1}^{1}= & 2 \sqrt{2} \bar{a} u_{\mathrm{m}} \exp \left(\frac{\bar{b}}{2 \bar{a}}\right) \\
\times & {\left[\exp \left(-\frac{\pi}{\bar{a}}\right)-\exp \left(-\frac{2 \pi}{\bar{a}}\right)\right] . }
\end{aligned}
$$

In order to show the contribution of the exponential term to the total time delay, we define the ratio $\eta_{2,1}$ as

$\eta_{2,1}=\log _{10}\left(\frac{\Delta T_{2,1}^{1}}{\Delta T_{2,1}}\right)$

The deviations of these observables in the strong deflection gravitational lensing by the quantum deformed Schwarzschild black hole from those of the Schwarzschild black hole can be worked out as 


$$
\begin{aligned}
\delta \theta_{\infty} & =\theta_{\infty}-\left.\theta_{\infty}\right|_{a=0}, \\
\delta s & =s-\left.s\right|_{a=0}, \\
\delta \Delta r & =\Delta r-\left.\Delta r\right|_{a=0}, \\
\delta \Delta T_{2,1} & =\Delta T_{2,1}-\left.\Delta T_{2,1}\right|_{a=0}, \\
\delta \eta_{2,1} & =\eta_{2,1}-\left.\eta_{2,1}\right|_{a=0} .
\end{aligned}
$$

\subsection{Example of Sgr A*}

Taking the supermassive black hole in the Galactic Center, Sgr A*, as a quantum deformed Schwarzschild black hole with the mass $m_{\bullet, \mathrm{SgrA} *}=4.28 \times 10^{6} \mathrm{M}_{\odot}$ and the distance $D_{\mathrm{OL}, \mathrm{SgrA} *}=8.32 \mathrm{kpc}$ [128], we estimate its observables in the strong deflection gravitational lensing on the domain $\mathcal{D}$ (8). The left panel of Fig. 4 shows its angular radius of the shadow $\theta_{\infty}$, angular separation $s$ and brightness difference $\Delta r$ between the first relativistic image and the packed others, differential time delay $\Delta T_{2,1}$ between the first and the second relativistic images and the ratio $\eta_{2,1}$ (see the left- $y$ axes); it also indicates the deviations of these observables from those of the Schwarzschild black hole (see the right- $y$ axes).

The angular radius of the shadow $\theta_{\infty}$ increases with the quantum deformation and changes from about 26 to $31 \mu$ as, which might be able to be observed with EHT [2]. Its deviation from the one of the Schwarzschild black hole $\delta \theta_{\infty}$ is no more than $5 \mu$ as, which is below the current ability, making it impossible to distinguish the quantum deformed Schwarzschild black hole from the Schwarzschild black hole by measuring $\delta \theta_{\infty}$. The angular separation between the first relativistic image and the packed others $s$ decreases with the quantum deformation $a$ from about 33 to 20 nas. Its absolute deviation from the one of the Schwarzschild black hole $\delta s$ is less than about 14 nas. Both $s$ and $\delta s$ are far beyond the reach of techniques in the near future. The brightness difference between the first relativistic image and the packed others $\Delta r$ grows with the quantum deformation from about 6.8 to 7.5 mag and its deviation from the one of the Schwarzschild black hole $\delta \Delta r$ is no more than $0.8 \mathrm{mag}$. Although $\Delta r$ and $\delta \Delta r$ are well within the current ability of photometry, neither of them can be measurable because the angular separation between them $s$ is too tiny to resolve. The differential time delay between the first and second relativistic images $\Delta T_{2,1}$ increases with respect to the quantum deformation from 11.6 to $13.6 \mathrm{~min}$, while its deviation from the one of the Schwarzschild black hole $\delta \Delta T_{2,1}$ is less than $2 \mathrm{~min}$. Both of them are shorter than the typical time span of an observational session of EHT, which is typical hours, and therefore cannot be detected. The ratio of the exponential term to the total time delay $\eta_{2,1}$ decreases with the quantum deformation $a$ from 1.5 to $1.0 \%$ and its absolute deviation from the one of the Schwarzschild black hole $\delta \eta_{2,1}$ is no more than $0.5 \%$. The inaccessibility of the differential time delay makes $\eta_{2,1}$ and $\delta \eta_{2,1}$ unmeasurable as well.
In summary for the case of Sgr $\mathrm{A}^{*}$, we find that (1) it is possible to measure the angular size of the shadow $\theta_{\infty}$ cast by the quantum deformed Schwarzschild black hole with EHT; (2) none of other observables of the relativistic images, including the angular separation $s$, the brightness difference $\Delta r$ and the time delay $\Delta T_{2,1}$, can be measured in the near future; and (3) it is currently impossible to distinguish the quantum deformed Schwarzschild black hole from the Schwarzschild black hole by measuring these observables in the strong deflection gravitational lensing.

For comparison, the renormalization group improved Schwarzschild black hole and the asymptotically safe black hole might have comparable and detectable angular size of their shadows as the one of the quantum deformed Schwarzschild black hole, while the absolute deviations of them from the one of the Schwarzschild black hole are no more than $4 \mu$ as [113] and $1.5 \mu$ as [107], respectively. The angular separations between the first relativistic image and the packed others for these two black holes can reach about 174 nas [113] and 87 nas, respectively, both of which are significantly bigger than the one of the quantum deformed Schwarzschild black hole, even though they are still too tiny to detect. It makes other observables relevant to the relativistic images undetectable for these two black holes as well. Therefore, in the case of Sgr A* as the lens, we find that these three kinds of the quantum-corrected black holes share almost the same detectability: it is currently impossible to distinguish them from the Schwarzschild black hole and from each other by the strong deflection gravitational lensing.

\subsection{Example of M87*}

Taking the supermassive black hole M87* as a quantum deformed Schwarzschild black hole with the mass of $m_{\bullet, \mathrm{M} 87 *}=6.5 \times 10^{9} \mathrm{M}_{\odot}$ and the distance of $D_{\mathrm{OL}, \mathrm{M} 87 *}=$ $16.8 \mathrm{Mpc}$ [7], we estimate its observables in the strong deflection gravitational lensing on the domain $\mathcal{D}(8)$. The right panel of Fig. 4 shows its angular radius of the shadow $\theta_{\infty}$, angular separation $s$, brightness difference $\Delta r$, differential time delay $\Delta T_{2,1}$ and ratio $\eta_{2,1}$ (see the left- $y$ axes) and their deviations from those of the Schwarzschild black hole (see the right- $y$ axes). All of the curves for M87* have the same shape as those of Sgr A*, whereas some of them have very different ranges since these two black holes have very different masses and distances even for a given dimensionless quantum deformation $a$.

For M87*, its apparent size of the shadow $\theta_{\infty}$ changes from about 20 to $23 \mu$ as, while its deviation from the one of the Schwarzschild black hole $\delta \theta_{\infty}$ is no more than $3.5 \mu$ as. The angular separation $s$ decreases from about 25 to 15 nas and its absolute deviation from the one of the Schwarzschild black hole $\delta s$ is less than 10 nas. The differential time delay $\Delta T_{2,1}$ and its deviation from the one of the Schwarzschild 


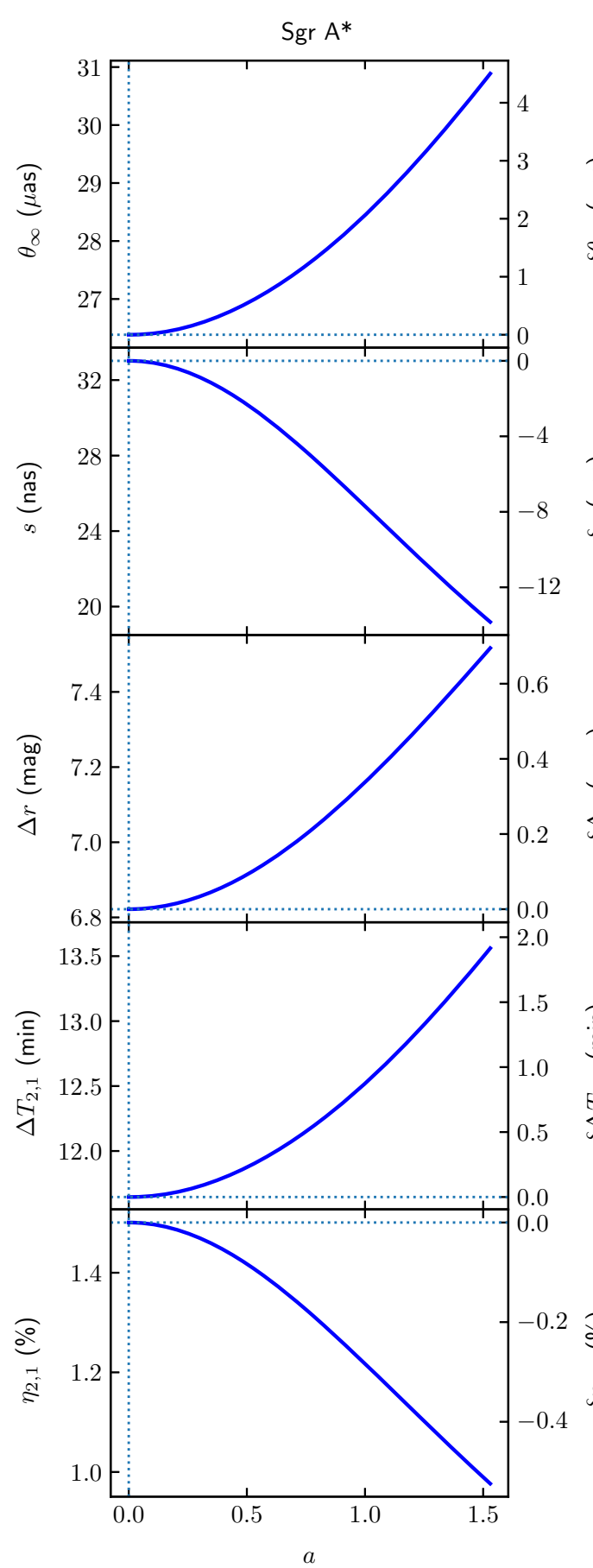

Fig. 4 The observables in the strong deflection gravitational lensing by the quantum deformed Schwarzschild black hole and their deviations from those of the Schwarzschild black hole are shown for Sgr A* (left panel) and M87* (right panel) on the domain $\mathcal{D}$. From top to bottom, the angular radius of the shadow $\theta_{\infty}$, the angular separation $s$

black hole can reach 14 and 2 days, respectively, both of which are significantly longer than those of Sgr A* due to M87*'s much bigger mass. However, neither of them are able to be measured since the angular separation between the first

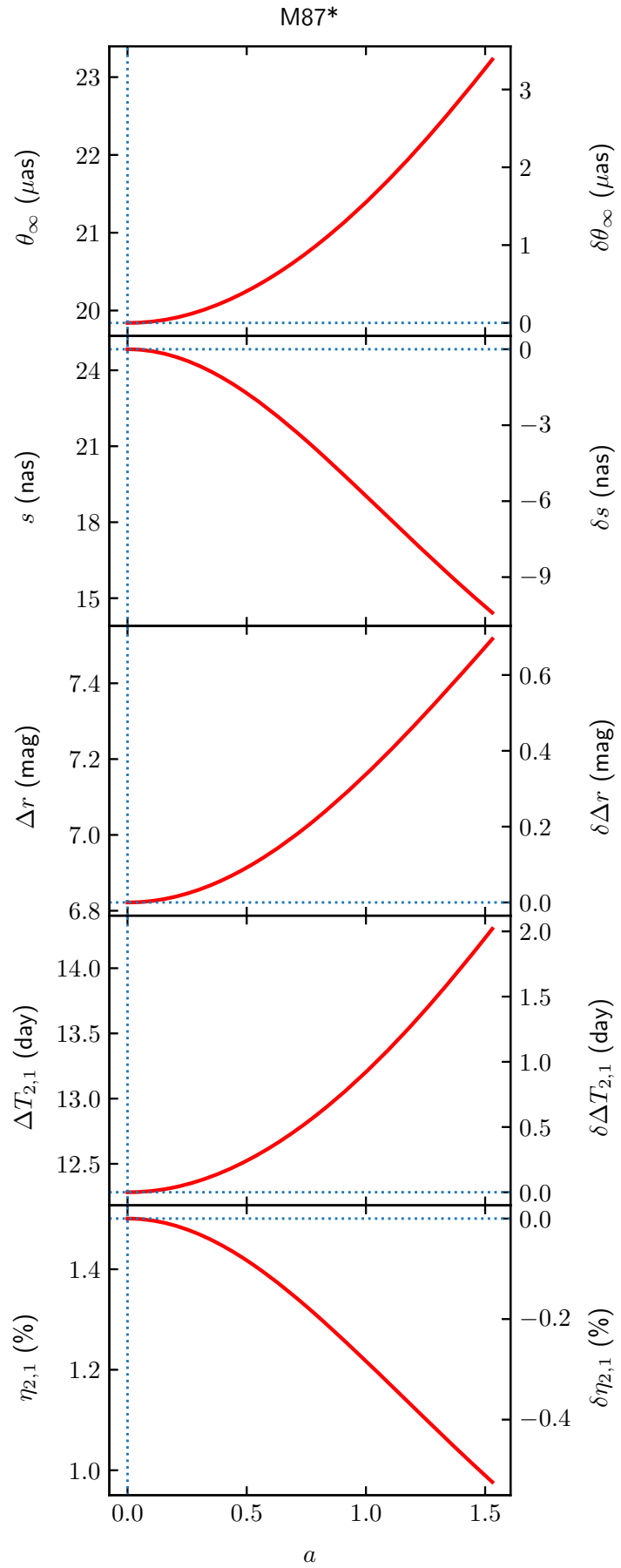

and brightness difference $\Delta r$ between the first relativistic image and the packed others, the differential time delay $\Delta T_{2,1}$ between the first and the second relativistic images and the ratio $\eta_{2,1}$ are demonstrated in the left- $y$ axes and the deviations of these observables from those of the Schwarzschild black hole are indicated in the right- $y$ axes

image and second one is too tiny to resolve. The brightness difference $\Delta r$ and the time delay ratio $\eta_{2,1}$ are the same as those of Sgr A*. 
In summary for M87*, we find that (1) it is possible to observe the apparent size of its shadow, (2) it is far beyond the reach of current techniques to measure other observables and all of the deviations due to extremely smallness of the angular separation $s$; and (3) it is impossible to distinguish the quantum deformed Schwarzschild black hole from the Schwarzschild black hole since all of the deviations of its observables are inaccessible.

For comparison in the case of $\mathrm{M} 87^{*}$, the renormalization group improved Schwarzschild black hole and the asymptotically safe black hole have comparable angular size of their shadows as the one of the quantum deformed Schwarzschild black hole, while the absolute deviations of them from the one of the Schwarzschild black hole are no more than $3 \mu$ as [113] and $1 \mu$ as [107], respectively. The angular separations between the first relativistic image and the packed others for these two black holes can reach about 105 nas [113] and 40 nas, respectively, but are still too tiny to detect, which makes other observables inaccessible. Thus, it is still impossible to distinguish these three kinds of quantum-corrected black holes from the Schwarzschild black hole and from each other through the strong deflection gravitational lensing by M87*.

\section{Conclusions and discussion}

In this work, we investigate the weak and strong deflection gravitational lensing by the quantum deformed Schwarzschild black hole. For the weak deflection gravitational lensing, we work out its weak deflection angle and lensing observables, such as the image positions, magnification and time delay between the two lensed images. Taking Sgr A* as the lens, we estimate their observability and find that it is possible to measure the angular separation, the angular difference, the fluxes difference and the time delay between the lensed images with current technology. The quantum deformed Schwarzschild black hole might be distinguished from the Schwarzschild black hole by measuring the deviation of the fluxes difference from the one of the Schwarzschild black hole during a quiet state of Sgr A* with a dedicated space telescope and by detecting the deviations of the angular separation, the angular difference and the time delay with GRAVITY+ in the near future. For the strong deflection gravitational lensing, we obtain its observables, including the apparent radius of the shadow, the angular separation and the brightness difference between the first and the packed other images, and the time delays between the first and second relativistic images. After taking $\mathrm{Sgr} \mathrm{A}^{*}$ and $\mathrm{M} 87^{*}$ as the lenses, we assess the possibility for detecting these observables and their deviations from those of the Schwarzschild black hole. We find that it is possible to measure the apparent size of the shadows cast by these two supermassive black holes, while no other observables and none of their deviations are well within the reach of current techniques. Therefore, it is presently infeasible to distinguish the quantum deformed Schwarzschild black hole from the Schwarzschild black hole by the observables in the strong deflection gravitational lensing. Nevertheless, we can still obtain a preliminary bound on the (dimensionless) quantum deformation as $0<a<1.53$ based on the apparent size of M87*'s shadow observed by EHT.

The observability of the quantum deformed Schwarzschild black hole is also intensively compared with those of the renormalization group improved Schwarzschild black hole and the asymptotically safe black hole. Contrary to the case of the quantum deformed Schwarzschild black hole, it is not possible to distinguish the renormalization group improved Schwarzschild black hole and the asymptotically safe black hole from the Schwarzschild black hole through the weak deflection gravitational lensing by Sgr A*. Meanwhile, these three kinds of quantum black holes cannot be distinguished from the Schwarzschild black hole and from each other through the strong deflection gravitational lensing by Sgr $\mathrm{A}^{*}$ and $\mathrm{M} 87^{*}$. Therefore, we find that it is most likely to test the quantum deformed Schwarzschild black hole by the weak deflection gravitational lensing in the foreseen future among these black holes.

In the Universe, black holes rotate. In fact, the observation by EHT disfavors a non-rotating black hole of M87* [2]. Gravitational lensing by a spinning quantum deformed black hole will be an interesting topic, however, to our best knowledge, the metric for such a rotating black hole with quantum fluctuations is still absent. Even so, we might catch a glimpse that its gravitational-lensing signatures would be very different from those presented in this work since the spin can make the caustic shifted and distorted based on previous studies in other scenarios [138-154]. Meanwhile, the interpretation of the apparent size and shape of M87* observed by EHT depends heavily on a bank of the general relativistic magnetohydrodynamics of plasma around the Kerr black hole [6], which adopts many untested assumptions about accretion flow and emission physics [155]. Any deviation in the spacetime caused by the quantum effects would not be told from the violation of these astrophysical assumptions. The photon ring [156] around $\mathrm{M} 87^{*}$ might provide a robust test, which, however, needs very challenging space-based interferometry [157-159]. Thus, we cannot constrain the quantum deformation based on the M87*'s shadow in a self-consistent manner for now, but the preliminary bound on it we obtain in this work might still be able to provide a hint of it with the same orderof-magnitude as the real one. Considering that it would not be easy to distinguish the quantum deformed Schwarzschild black hole from the Schwarzschild black hole, the timelike geodesic motions around it, such as precessing [160-166] and periodic [167-175] orbits, might be other important way to investigate its quantum effects. 
Acknowledgements This work is funded by the National Natural Science Foundation of China (Grant nos. 11573015 and 11833004).

Data Availability Statement This manuscript has no associated data or the data will not be deposited. [Authors' comment: This paper is a theoretical work and all of the data are adopted by the related references.]

Open Access This article is licensed under a Creative Commons Attribution 4.0 International License, which permits use, sharing, adaptation, distribution and reproduction in any medium or format, as long as you give appropriate credit to the original author(s) and the source, provide a link to the Creative Commons licence, and indicate if changes were made. The images or other third party material in this article are included in the article's Creative Commons licence, unless indicated otherwise in a credit line to the material. If material is not included in the article's Creative Commons licence and your intended use is not permitted by statutory regulation or exceeds the permitted use, you will need to obtain permission directly from the copyright holder. To view a copy of this licence, visit http://creativecomm ons.org/licenses/by/4.0/.

Funded by SCOAP ${ }^{3}$.

\section{Appendix A: Renormalization group improved Schwarzschild and asymptotically safe black holes}

The metric of renormalization group improved Schwarzschild black hole reads [41]

$$
\begin{aligned}
A(r) & =[B(r)]^{-1} \\
& =1-\frac{2 m \bullet}{r}\left(1+\Omega \frac{m_{\bullet}^{2}}{r^{2}}+\gamma \Omega \frac{m_{\bullet}^{3}}{r^{3}}\right)^{-1}, \\
C(r) & =r^{2},
\end{aligned}
$$

where $\gamma$ and $\Omega$ are two positive constants related to the identification of cutoff of the distance scale and from the nonperturbative renormalization group theory. Its expanded form in the weak field approximation is

$$
\begin{aligned}
A(r)= & 1-2 \frac{m_{\bullet}}{r}+2 \Omega \frac{m_{\bullet}^{3}}{r^{3}}+2 \gamma \Omega \frac{m_{\bullet}^{4}}{r^{4}}+\mathcal{O}\left(\frac{m_{\bullet}^{5}}{r^{5}}\right), \\
B(r)= & 1+2 \frac{m_{\bullet}}{r}+4 \frac{m_{\bullet}^{2}}{r^{2}}+(-2 \Omega+8) \frac{m_{\bullet}^{3}}{r^{3}} \\
& +(-2 \gamma \Omega+8 \Omega+16) \frac{m_{\bullet}^{4}}{r^{4}}+\mathcal{O}\left(\frac{m_{\bullet}^{5}}{r^{5}}\right) .
\end{aligned}
$$

It is clear that the quantum improvement starts to appear in the third-order term in $A(r)$ and $B(r)$.

The metric of the asymptotically safe black hole reads [107]

$$
\begin{aligned}
& A(r)=[B(r)]^{-1}=1-\frac{2 m_{\bullet}}{r}\left(1+\Gamma \frac{m_{\bullet}^{3}}{r^{3}}\right)^{-1}, \\
& C(r)=r^{2},
\end{aligned}
$$

where $\Gamma$ is a positive constant no more than $32 / 27$. Its expanded form in the weak field approximation is

$$
\begin{aligned}
A(r)= & 1-2 \frac{m_{\bullet}}{r}+2 \Gamma \frac{m_{\bullet}^{4}}{r^{4}}+\mathcal{O}\left(\frac{m_{\bullet}^{7}}{r^{7}}\right), \\
B(r)= & 1+2 \frac{m \bullet}{r}+4 \frac{m_{\bullet}^{2}}{r^{2}}+8 \frac{m_{\bullet}^{3}}{r^{3}} \\
& +(16-2 \Gamma) \frac{m_{\bullet}^{4}}{r^{4}}+(32-8 \Gamma) \frac{m_{\bullet}^{5}}{r^{5}} \\
& +(64-24 \Gamma) \frac{m_{\bullet}^{6}}{r^{6}}+\mathcal{O}\left(\frac{m_{\bullet}^{7}}{r^{7}}\right) .
\end{aligned}
$$

The quantum correction $\Gamma$ starts to play a role in the fourthorder term in $A(r)$ and $B(r)$.

\section{References}

1. C.M. Will, Living Rev. Relativ. 17, 4 (2014). https://doi.org/10. 12942/lrr-2014-4

2. Event Horizon Telescope Collaboration, Astrophys. J. Lett. 875, L1 (2019). https://doi.org/10.3847/2041-8213/ab0ec7

3. Event Horizon Telescope Collaboration, Astrophys. J. Lett. 875, L2 (2019). https://doi.org/10.3847/2041-8213/ab0c96

4. Event Horizon Telescope Collaboration, Astrophys. J. Lett. 875, L3 (2019). https://doi.org/10.3847/2041-8213/ab0c57

5. Event Horizon Telescope Collaboration, Astrophys. J. Lett. 875, L4 (2019). https://doi.org/10.3847/2041-8213/ab0e85

6. Event Horizon Telescope Collaboration, Astrophys. J. Lett. 875, L5 (2019). https://doi.org/10.3847/2041-8213/ab0f43

7. Event Horizon Telescope Collaboration, Astrophys. J. Lett. 875, L6 (2019). https://doi.org/10.3847/2041-8213/ab1141

8. LIGO Scientific Collaboration and Virgo Collaboration, Phys. Rev. Lett. 116(6), 061102 (2016). https://doi.org/10.1103/ PhysRevLett.116.061102

9. LIGO Scientific Collaboration and Virgo Collaboration, Phys. Rev. X 6(4), 041015 (2016). https://doi.org/10.1103/PhysRevX. 6.041015

10. LIGO Scientific Collaboration and Virgo Collaboration, Phys. Rev. Lett. 116(24), 241103 (2016). https://doi.org/10.1103/ PhysRevLett.116.241103

11. LIGO Scientific Collaboration and Virgo Collaboration, Phys. Rev. Lett. 118(22), 221101 (2017). https://doi.org/10.1103/ PhysRevLett.118.221101

12. LIGO Scientific Collaboration and Virgo Collaboration, Astrophys. J. Lett. 851, L35 (2017). https://doi.org/10.3847/ 2041-8213/aa9f0c

13. LIGO Scientific Collaboration and Virgo Collaboration, Phys. Rev. Lett. 119(14), 141101 (2017). https://doi.org/10.1103/ PhysRevLett.119.141101

14. J. Bardeen, in Proceedings of International Conference GR5 (Tbilisi University Press, Tbilisi, USSR, 1968), p. 174

15. S.A. Hayward, Phys. Rev. Lett. 96(3), 031103 (2006). https://doi. org/10.1103/PhysRevLett.96.031103

16. C. Bejarano, G.J. Olmo, D. Rubiera-Garcia, Phys. Rev. D 95(6), 064043 (2017). https://doi.org/10.1103/PhysRevD.95.064043

17. C.C. Menchon, G.J. Olmo, D. Rubiera-Garcia, Phys. Rev. D 96(10), 104028 (2017). https://doi.org/10.1103/PhysRevD.96. 104028

18. V.P. Frolov, G.A. Vilkovisky, Phys. Lett. B 106, 307 (1981). https://doi.org/10.1016/0370-2693(81)90542-6 
19. M. Ambrus, P. Hájíček, Phys. Rev. D 72(6), 064025 (2005). https://doi.org/10.1103/PhysRevD.72.064025

20. C. Rovelli, F. Vidotto, Int. J. Mod. Phys. D 23(12), 1442026 (2014). https://doi.org/10.1142/S0218271814420267

21. C. Barceló, S. Liberati, S. Sonego, M. Visser, Phys. Rev. D 77(4), 044032 (2008). https://doi.org/10.1103/PhysRevD.77.044032

22. S.D. Mathur, Class. Quantum Gravity 26(22), 224001 (2009). https://doi.org/10.1088/0264-9381/26/22/224001

23. S.D. Mathur, D. Turton, J. High Energy Phys. 01, 34 (2014). https://doi.org/10.1007/JHEP01(2014)034

24. B. Guo, S. Hampton, S.D. Mathur, J. High Energy Phys. 07, 162 (2018). https://doi.org/10.1007/JHEP07(2018)162

25. R. Carballo-Rubio, F. Di Filippo, S. Liberati, M. Visser, Phys. Rev. D 98(12), 124009 (2018). https://doi.org/10.1103/PhysRevD.98. 124009

26. V. Cardoso, P. Pani, Nat. Astron. 1, 586 (2017). https://doi.org/ 10.1038/s41550-017-0225-y

27. S.B. Giddings, S. Koren, G. Treviño, Phys. Rev. D 100(4), 044005 (2019). https://doi.org/10.1103/PhysRevD.100.044005

28. V. Cardoso, P. Pani, Living Rev. Relativ. 22(1), 4 (2019). https:// doi.org/10.1007/s41114-019-0020-4

29. Z. Bern, Living Rev. Relativ. 5(1), 5 (2002). https://doi.org/10. 12942/lrr-2002-5

30. C.P. Burgess, Living Rev. Relativ. (2004). https://doi.org/10. 12942/lrr-2004-5

31. M. Bojowald, Living Rev. Relativ. 8, 11 (2005). https://doi.org/ 10.1007/lrr-2005-11

32. M. Niedermaier, M. Reuter, Living Rev. Relativ. 9(1), 5 (2006). https://doi.org/10.12942/lrr-2006-5

33. C. Rovelli, Living Rev. Relativ. 11(1), 5 (2008). https://doi.org/ 10.12942/lrr-2008-5

34. S. Hossenfelder, Living Rev. Relativ. 16, 2 (2013). https://doi.org/ 10.12942/lrr-2013-2

35. A. Perez, Living Rev. Relativ. 16(1), 3 (2013). https://doi.org/10. 12942/1rr-2013-3

36. G. Amelino-Camelia, Living Rev. Relativ. 16, 5 (2013). https:// doi.org/10.12942/lrr-2013-5

37. J.F. Donoghue, B.R. Holstein, B. Garbrecht, T. Konstandin, Phys. Lett. B 529(1-2), 132 (2002). https://doi.org/10.1016/ S0370-2693(02)01246-7

38. N.E. Bjerrum-Bohr, J.F. Donoghue, B.R. Holstein, Phys. Rev. D 68(8), 084005 (2003). https://doi.org/10.1103/PhysRevD.68. 084005

39. G.G. Kirilin, Phys. Rev. D 75(10), 108501 (2007). https://doi.org/ 10.1103/PhysRevD.75.108501

40. A. Bonanno, M. Reuter, Phys. Rev. D 60, 084011 (1999). https:// doi.org/10.1103/PhysRevD.60.084011

41. A. Bonanno, M. Reuter, Phys. Rev. D 62(4), 043008 (2000). https://doi.org/10.1103/PhysRevD.62.043008

42. A. Bonanno, M. Reuter, Phys. Rev. D 73, 083005 (2006). https:// doi.org/10.1103/PhysRevD.73.083005

43. M. Reuter, E. Tuiran, Phys. Rev. D 83(4), 044041 (2011). https:// doi.org/10.1103/PhysRevD.83.044041

44. R. Gambini, J. Pullin, Phys. Rev. Lett. 101, 161301 (2008). https:// doi.org/10.1103/PhysRevLett.101.161301

45. R. Gambini, J. Pullin, Phys. Rev. Lett. 110, 211301 (2013). https:// doi.org/10.1103/PhysRevLett.110.211301

46. P. Nicolini, A. Smailagic, E. Spallucci, Phys. Lett. B 632(4), 547 (2006). https://doi.org/10.1016/j.physletb.2005.11.004

47. S. Ansoldi, P. Nicolini, A. Smailagic, E. Spallucci, Phys. Lett. B 645(2-3), 261 (2007). https://doi.org/10.1016/j.physletb.2006. 12.020

48. E. Spallucci, A. Smailagic, P. Nicolini, Phys. Lett. B 670(4-5), 449 (2009). https://doi.org/10.1016/j.physletb.2008.11.030

49. P. Nicolini, Int. J. Mod. Phys. A 24(07), 1229 (2009). https://doi. org/10.1142/S0217751X09043353
50. B.J. Carr, Mod. Phys. Lett. A 28, 1340011 (2013). https://doi.org/ 10.1142/S0217732313400117

51. J.R. Mureika, Phys. Lett. B 789, 88 (2019). https://doi.org/10. 1016/j.physletb.2018.12.009

52. D.I. Kazakov, S.N. Solodukhin, Nucl. Phys. B 429(1), 153 (1994). https://doi.org/10.1016/S0550-3213(94)80045-6

53. S.N. Solodukhin, Phys. Rev. D 51(2), 618 (1995). https://doi.org/ 10.1103/PhysRevD.51.618

54. M. Saleh, B.B. Thomas, T.C. Kofane, Astrophys. Space Sci. 350(2), 721 (2014). https://doi.org/10.1007/s10509-013-1761-2

55. M. Shahjalal, Nucl. Phys. B 940, 63 (2019). https://doi.org/10. 1016/j.nuclphysb.2019.01.009

56. S. Eslamzadeh, K. Nozari, Nucl. Phys. B 959, 115136 (2020). https://doi.org/10.1016/j.nuclphysb.2020.115136

57. W. Kim, Y. Kim, Phys. Lett. B 718(2), 687 (2012). https://doi. org/10.1016/j.physletb.2012.11.017

58. R.A. Konoplya, Phys. Lett. B 804, 135363 (2020). https://doi.org/ 10.1016/j.physletb.2020.135363

59. J. Peng, M. Guo, X.H. Feng (2020), arXiv e-prints. arXiv:2008.00657

60. V. Perlick, Living Rev. Relativ. 7, 9 (2004). https://doi.org/10. 12942/lrr-2004-9

61. P. Schneider, J. Ehlers, E.E. Falco, Gravitational Lenses (Springer, Berlin, 1992). https://doi.org/10.1007/978-3-662-03758-4

62. A.O. Petters, H. Levine, J. Wambsganss, Singularity Theory and Gravitational Lensing (Birkhäuser, Boston, 2001). https://doi. org/10.1007/978-1-4612-0145-8

63. P. Schneider, C.S. Kochanek, J. Wambsganss, in Saas-Fee Advanced Course 33: Gravitational Lensing: Strong, Weak and Micro, ed. by G. Meylan, P. Jetzer, P. North (Springer, Berlin, 2006). https://doi.org/10.1007/978-3-540-30310-7

64. K.C. Sahu, J. Anderson, S. Casertano, H.E. Bond, P. Bergeron, E.P. Nelan, L. Pueyo, T.M. Brown, A. Bellini, Z.G. Levay, J. Sokol, M. Dominik, A. Calamida, N. Kains, M. Livio, Science 356, 1046 (2017). https://doi.org/10.1126/science.aal2879

65. C.R. Keeton, A.O. Petters, Phys. Rev. D 72(10), 104006 (2005). https://doi.org/10.1103/PhysRevD.72.104006

66. C.R. Keeton, A.O. Petters, Phys. Rev. D 73(4), 044024 (2006) https://doi.org/10.1103/PhysRevD.73.044024

67. C.R. Keeton, A.O. Petters, Phys. Rev. D 73(10), 104032 (2006). https://doi.org/10.1103/PhysRevD.73.104032

68. W.G. Cao, Y. Xie, Eur. Phys. J. C 78, 191 (2018). https://doi.org/ 10.1140/epjc/s10052-018-5684-5

69. X.T. Cheng, Y. Xie, Phys. Rev. D 103(6), 064040 (2021). https:// doi.org/10.1103/PhysRevD.103.064040

70. C. Darwin, Proc. R. Soc. A 249, 180 (1959). https://doi.org/10. 1098/rspa.1959.0015

71. K.S. Virbhadra, D. Narasimha, S.M. Chitre, Astron. Astrophys. 337, 1 (1998)

72. K.S. Virbhadra, G.F.R. Ellis, Phys. Rev. D 62(8), 084003 (2000)

73. V. Bozza, Phys. Rev. D 66(10), 103001 (2002). https://doi.org/ 10.1103/PhysRevD.66.103001

74. E.F. Eiroa, G.E. Romero, D.F. Torres, Phys. Rev. D 66(2), 024010 (2002). https://doi.org/10.1103/PhysRevD.66.024010

75. V. Bozza, Phys. Rev. D 67(10), 103006 (2003). https://doi.org/ 10.1103/PhysRevD.67.103006

76. S.E. Vázquez, E.P. Esteban, Nuovo Cimento B Ser. 119, 489 (2004). https://doi.org/10.1393/ncb/i2004-10121-y

77. N. Tsukamoto, Phys. Rev. D 95(6), 064035 (2017). https://doi. org/10.1103/PhysRevD.95.064035

78. T. Hsieh, D.S. Lee, C.Y. Lin, Phys. Rev. D 103(10), 104063 (2021). https://doi.org/10.1103/PhysRevD.103.104063

79. A.Y. Bin-Nun, Phys. Rev. D 81(12), 123011 (2010). https://doi. org/10.1103/PhysRevD.81.123011

80. S.S. Zhao, Y. Xie, J. Cosmol. Astropart. Phys. 07, 007 (2016). https://doi.org/10.1088/1475-7516/2016/07/007 
81. X. Lu, F.W. Yang, Y. Xie, Eur. Phys. J. C 76, 357 (2016). https:// doi.org/10.1140/epjc/s10052-016-4218-2

82. S.S. Zhao, Y. Xie, Eur. Phys. J. C 77, 272 (2017). https://doi.org/ 10.1140/epjc/s10052-017-4850-5

83. S.S. Zhao, Y. Xie, Phys. Lett. B 774, 357 (2017). https://doi.org/ 10.1016/j.physletb.2017.09.090

84. S. Chakraborty, S. SenGupta, J. Cosmol. Astropart. Phys. 7, 045 (2017). https://doi.org/10.1088/1475-7516/2017/07/045

85. J.G. Cramer, R.L. Forward, M.S. Morris, M. Visser, G. Benford, G.A. Landis, Phys. Rev. D 51(6), 3117 (1995). https://doi.org/10. 1103/PhysRevD.51.3117

86. K.K. Nandi, Y.Z. Zhang, A.V. Zakharov, Phys. Rev. D 74(2), 024020 (2006). https://doi.org/10.1103/PhysRevD.74.024020

87. C. Bambi, Phys. Rev. D 87(10), 107501 (2013). https://doi.org/ 10.1103/PhysRevD.87.107501

88. K.K. Nandi, R.N. Izmailov, E.R. Zhdanov, A. Bhattacharya, J. Cosmol. Astropart. Phys. 2018(7), 027 (2018). https://doi.org/10. 1088/1475-7516/2018/07/027

89. K. Jusufi, N. Sarkar, F. Rahaman, A. Banerjee, S. Hansraj, Eur. Phys. J. C 78(4), 349 (2018). https://doi.org/10.1140/epjc/ s10052-018-5823-z

90. K. Jusufi, A. Övgün, Phys. Rev. D 97(2), 024042 (2018). https:// doi.org/10.1103/PhysRevD.97.024042

91. R. Shaikh, Phys. Rev. D 98(2), 024044 (2018). https://doi.org/10. 1103/PhysRevD.98.024044

92. R. Shaikh, P. Banerjee, S. Paul, T. Sarkar, Phys. Lett. B 789, 270 (2019). https://doi.org/10.1016/j.physletb.2018.12.030

93. R. Shaikh, P. Banerjee, S. Paul, T. Sarkar, J. Cosmol. Astropart. Phys. 2019(7), 028 (2019). https://doi.org/10.1088/1475-7516/ 2019/07/028

94. M. Amir, K. Jusufi, A. Banerjee, S. Hansraj, Class. Quantum Gravity 36(21), 215007 (2019). https://doi.org/10.1088/1361-6382/ ab42be

95. S. Sahu, M. Patil, D. Narasimha, P.S. Joshi, Phys. Rev. D 86(6), 063010 (2012). https://doi.org/10.1103/PhysRevD.86.063010

96. R. Shaikh, P. Kocherlakota, R. Narayan, P.S. Joshi, Mon. Not. R. Astron. Soc. 482(1), 52 (2019). https://doi.org/10.1093/mnras/ sty 2624

97. T. Kubo, N. Sakai, Phys. Rev. D 93(8), 084051 (2016). https:// doi.org/10.1103/PhysRevD.93.084051

98. P.V.P. Cunha, J.A. Font, C. Herdeiro, E. Radu, N. Sanchis-Gual, M. Zilhao, Phys. Rev. D 96(10), 104040 (2017). https://doi.org/ 10.1103/PhysRevD.96.104040

99. M. Patil, P. Mishra, D. Narasimha, Phys. Rev. D 95(2), 024026 (2017). https://doi.org/10.1103/PhysRevD.95.024026

100. R. Shaikh, P. Banerjee, S. Paul, T. Sarkar, Phys. Rev. D 99(10), 104040 (2019). https://doi.org/10.1103/PhysRevD.99.104040

101. X.Y. Zhu, Y. Xie, Eur. Phys. J. C 80(5), 444 (2020). https://doi. org/10.1140/epjc/s10052-020-8021-8

102. N.E.J. Bjerrum-Bohr, J.F. Donoghue, B.R. Holstein, L. Planté, P. Vanhove, Phys. Rev. Lett. 114(6), 061301 (2015). https://doi.org/ 10.1103/PhysRevLett.114.061301

103. S. Haroon, M. Jamil, K. Lin, P. Pavlovic, M. Sossich, A. Wang, Eur. Phys. J. C 78(6), 519 (2018). https://doi.org/10.1140/epjc/ s10052-018-5986-7

104. X. Li, Z. Chang, Phys. Rev. D 82(12), 124009 (2010). https://doi. org/10.1103/PhysRevD.82.124009

105. C. Xu, Y. Yang, Int. J. Mod. Phys. A 35(29), 2050188-52 (2020). https://doi.org/10.1142/S0217751X20501882

106. H. Ghaffarnejad, M.A. Mojahedi, Res. Astron. Astrophys. 17, 052 (2017). https://doi.org/10.1088/1674-4527/17/6/52

107. A. Held, R. Gold, A. Eichhorn, J. Cosmol. Astropart. Phys. 2019(6), 029 (2019). https://doi.org/10.1088/1475-7516/2019/ $06 / 029$
108. E. Contreras, Á. Rincón, G. Panotopoulos, P. Bargueño, B. Koch, Phys. Rev. D 101(6), 064053 (2020). https://doi.org/10.1103/ PhysRevD.101.064053

109. C. Liu, T. Zhu, Q. Wu, K. Jusufi, M. Jamil, M. Azreg-Aïnou, A. Wang, Phys. Rev. D 101(8), 084001 (2020). https://doi.org/10. 1103/PhysRevD.101.084001

110. C. Ding, S. Kang, C.Y. Chen, S. Chen, J. Jing, Phys. Rev. D 83(8), 084005 (2011). https://doi.org/10.1103/PhysRevD.83.084005

111. S.W. Wei, P. Cheng, Y. Zhong, X.N. Zhou, J. Cosmol. Astropart. Phys. 2015(8), 004 (2015). https://doi.org/10.1088/1475-7516/ 2015/08/004

112. M. Sharif, S. Iftikhar, Eur. Phys. J. C 76(11), 630 (2016). https:// doi.org/10.1140/epjc/s10052-016-4472-3

113. X. Lu, Y. Xie, Eur. Phys. J. C 79(12), 1016 (2019). https://doi. org/10.1140/epjc/s10052-019-7537-2

114. R. Kumar, B.P. Singh, S.G. Ghosh, Ann. Phys. 420, 168252 (2020). https://doi.org/10.1016/j.aop.2020.168252

115. S. Sahu, K. Lochan, D. Narasimha, Phys. Rev. D 91(6), 063001 (2015). https://doi.org/10.1103/PhysRevD.91.063001

116. X. Lu, Y. Xie, Mod. Phys. Lett. A 34(20), 1950152 (2019). https:// doi.org/10.1142/S0217732319501529

117. A.F. Grillo, E. Luzio, F. Méndez, F. Torres, Int. J. Mod. Phys. D 21, 1250007 (2012). https://doi.org/10.1142/S0218271812500071

118. Z. Horváth, L.Á. Gergely, Z. Keresztes, T. Harko, F.S.N. Lobo, Phys. Rev. D 84(8), 083006 (2011). https://doi.org/10.1103/ PhysRevD.84.083006

119. E.F. Eiroa, C.M. Sendra, Phys. Rev. D 86(8), 083009 (2012). https://doi.org/10.1103/PhysRevD.86.083009

120. R.N. Izmailov, R.K. Karimov, E.R. Zhdanov, K.K. Nandi, Mon. Not. R. Astron. Soc. 483(3), 3754 (2019). https://doi.org/10.1093/ mnras/sty3350

121. C.Y. Wang, Y.F. Shen, Y. Xie, J. Cosmol. Astropart. Phys. 04, 022 (2019). https://doi.org/10.1088/1475-7516/2019/04/022

122. F.Y. Liu, Y.F. Mai, W.Y. Wu, Y. Xie, Phys. Lett. B 795, 475 (2019). https://doi.org/10.1016/j.physletb.2019.06.052

123. X. Lu, Y. Xie, Eur. Phys. J. C 80(7), 625 (2020). https://doi.org/ 10.1140/epjc/s10052-020-8205-2

124. Y.X. Gao, Y. Xie, Phys. Rev. D 103, 043008 (2021). https://doi. org/10.1103/PhysRevD.103.043008

125. Event Horizon Telescope Collaboration, Phys. Rev. Lett. 125, 141104 (2020). https://doi.org/10.1103/PhysRevLett.125. 141104

126. S. Weinberg, Gravitation and Cosmology: Principles and Applications of the General Theory of Relativity (Wiley, New York, 1972)

127. S. Refsdal, Mon. Not. R. Astron. Soc. 128, 295 (1964). https:// doi.org/10.1093/mnras/128.4.295

128. S. Gillessen, P.M. Plewa, F. Eisenhauer, R. Sari et al., Astrophys. J. 837, 30 (2017). https://doi.org/10.3847/1538-4357/aa5c41

129. GRAVITY Collaboration, Astron. Astrophys. 602, A94 (2017). https://doi.org/10.1051/0004-6361/201730838

130. GRAVITY Collaboration, Astron. Astrophys. 647, A59 (2021). https://doi.org/10.1051/0004-6361/202040208

131. C.X. Huang, J. Burt, A. Vanderburg, M.N. Günther, A. Shporer, J.A. Dittmann, J.N. Winn, R. Wittenmyer, L. Sha, S.R. Kane, G.R. Ricker, R.K. Vanderspek, D.W. Latham, S. Seager, J.M. Jenkins, D.A. Caldwell, K.A. Collins, N. Guerrero, J.C. Smith, S.N. Quinn, S. Udry, F. Pepe, F. Bouchy, D. Ségransan, C. Lovis, D. Ehrenreich, M. Marmier, M. Mayor, B. Wohler, K. Haworth, E.H. Morgan, M. Fausnaugh, D.R. Ciardi, J. Christiansen, D. Charbonneau, D. Dragomir, D. Deming, A. Glidden, A.M. Levine, P.R. McCullough, L. Yu, N. Narita, T. Nguyen, T. Morton, J. Pepper, A. Pál, J.E. Rodriguez, K.G. Stassun, G. Torres, A. Sozzetti, J.P. Doty, J. Christensen-Dalsgaard, G. Laughlin, M. Clampin, J.L. Bean, L.A. Buchhave, G.Á. Bakos, B. Sato, S. Ida, L. Kaltenegger, E. Palle, D. Sasselov, R.P. Butler, J. Lissauer, J. Ge, S.A. 
Rinehart, Astrophys. J. Lett. 868, L39 (2018). https://doi.org/10. 3847/2041-8213/aaef91

132. GRAVITY Collaboration, Astron. Astrophys. 615, L15 (2018). https://doi.org/10.1051/0004-6361/201833718

133. GRAVITY Collaboration, Astron. Astrophys. 636, L5 (2020). https://doi.org/10.1051/0004-6361/202037813

134. C.M. Claudel, K.S. Virbhadra, G.F.R. Ellis, J. Math. Phys. 42, 818 (2001). https://doi.org/10.1063/1.1308507

135. D. Psaltis, F. Özel, C.K. Chan, D.P. Marrone, Astrophys. J. 814, 115 (2015). https://doi.org/10.1088/0004-637X/814/2/115

136. V. Bozza, S. Capozziello, G. Iovane, G. Scarpetta, Gen. Relativ. Gravit. 33, 1535 (2001). https://doi.org/10.1023/A: 1012292927358

137. V. Bozza, L. Mancini, Gen. Relativ. Gravit. 36, 435 (2004). https:// doi.org/10.1023/B:GERG.0000010486.58026.4f

138. J. Ibanez, Astron. Astrophys. 124, 175 (1983)

139. I. Bray, Phys. Rev. D 34, 367 (1986). https://doi.org/10.1103/ PhysRevD.34.367

140. S.A. Klioner, Sov. Astron. 35, 523 (1991)

141. J.F. Glicenstein, Astron. Astrophys. 343, 1025 (1999)

142. M. Sereno, F. De Luca, Phys. Rev. D 74(12), 123009 (2006). https://doi.org/10.1103/PhysRevD.74.123009

143. M.C. Werner, A.O. Petters, Phys. Rev. D 76(6), 064024 (2007). https://doi.org/10.1103/PhysRevD.76.064024

144. M. Sereno, F. De Luca, Phys. Rev. D 78(2), 023008 (2008). https:// doi.org/10.1103/PhysRevD.78.023008

145. A.B. Aazami, C.R. Keeton, A.O. Petters, J. Math. Phys. 52(9), 092502 (2011). https://doi.org/10.1063/1.3642614

146. A.B. Aazami, C.R. Keeton, A.O. Petters, J. Math. Phys. 52(10), 102501 (2011). https://doi.org/10.1063/1.3642616

147. G. He, W. Lin, Int. J. Mod. Phys. D 23, 1450031 (2014). https:// doi.org/10.1142/S021827181450031X

148. G. He, C. Jiang, W. Lin, Int. J. Mod. Phys. D 23, 1450079 (2014). https://doi.org/10.1142/S0218271814500795

149. X.M. Deng, Int. J. Mod. Phys. D 24, 1550056 (2015). https://doi. org/10.1142/S021827181550056X

150. G.S. He, W.B. Lin, Res. Astron. Astrophys. 15, 646 (2015). https:// doi.org/10.1088/1674-4527/15/5/003

151. X.M. Deng, Int. J. Mod. Phys. D 25, 1650082 (2016). https://doi. org/10.1142/S0218271816500826

152. G. He, W. Lin, Phys. Rev. D 93(2), 023005 (2016). https://doi. org/10.1103/PhysRevD.93.023005

153. G. He, W. Lin, Phys. Rev. D 94(6), 063011 (2016). https://doi. org/10.1103/PhysRevD.94.063011

154. G. He, W. Lin, Class. Quantum Gravity 34(10), 105006 (2017). https://doi.org/10.1088/1361-6382/aa691d

155. S.E. Gralla, Phys. Rev. D 103, 024023 (2021). https://doi.org/10. 1103/PhysRevD.103.024023
156. S.E. Gralla, D.E. Holz, R.M. Wald, Phys. Rev. D 100(2), 024018 (2019). https://doi.org/10.1103/PhysRevD.100.024018

157. M.D. Johnson, A. Lupsasca, A. Strominger, G.N. Wong, S. Hadar, D. Kapec, R. Narayan, A. Chael, C.F. Gammie, P. Galison, D.C.M. Palumbo, S.S. Doeleman, L. Blackburn, M. Wielgus, D.W. Pesce, J.R. Farah, J.M. Moran, Sci. Adv. 6(12), eaaz1310 (2020). https:// doi.org/10.1126/sciadv.aaz1310

158. S.E. Gralla, A. Lupsasca, Phys. Rev. D 102, 124003 (2020). https://doi.org/10.1103/PhysRevD.102.124003

159. S.E. Gralla, A. Lupsasca, D.P. Marrone, Phys. Rev. D 102, 124004 (2020). https://doi.org/10.1103/PhysRevD.102.124004

160. M. De Laurentis, R. De Rosa, F. Garufi, L. Milano, Mon. Not. R. Astron. Soc. 424, 2371 (2012). https://doi.org/10.1111/j. 1365-2966.2012.21410.x

161. X.M. Deng, Europhys. Lett. 120(6), 60004 (2017). https://doi. org/10.1209/0295-5075/120/60004

162. X.M. Deng, Eur. Phys. J. Plus 132, 85 (2017). https://doi.org/10. 1140/epjp/i2017-11376-1

163. I. De Martino, R. Lazkoz, M. De Laurentis, Phys. Rev. D 97(10), 104067 (2018). https://doi.org/10.1103/PhysRevD.97.104067

164. M. De Laurentis, I. De Martino, R. Lazkoz, Phys. Rev. D 97(10), 104068 (2018). https://doi.org/10.1103/PhysRevD.97.104068

165. M. De Laurentis, I. De Martino, R. Lazkoz, Eur. Phys. J. C 78(11), 916 (2018). https://doi.org/10.1140/epjc/s10052-018-6401-0

166. T.Y. Zhou, Y. Xie, Eur. Phys. J. C 80(11), 1070 (2020). https:// doi.org/10.1140/epjc/s10052-020-08661-w

167. J. Levin, G. Perez-Giz, Phys. Rev. D 77(10), 103005 (2008). https://doi.org/10.1103/PhysRevD.77.103005

168. J. Levin, R. Grossman, Phys. Rev. D 79(4), 043016 (2009). https:// doi.org/10.1103/PhysRevD.79.043016

169. J. Levin, G. Perez-Giz, Phys. Rev. D 79(12), 124013 (2009). https://doi.org/10.1103/PhysRevD.79.124013

170. J. Levin, Class. Quantum Gravity 26(23), 235010 (2009). https:// doi.org/10.1088/0264-9381/26/23/235010

171. V. Misra, J. Levin, Phys. Rev. D 82(8), 083001 (2010). https:// doi.org/10.1103/PhysRevD.82.083001

172. B. Gao, X.M. Deng, Ann. Phys. 418, 168194 (2020). https://doi. org/10.1016/j.aop.2020.168194

173. X.M. Deng, Eur. Phys. J. C 80(6), 489 (2020). https://doi.org/10. 1140/epjc/s10052-020-8067-7

174. X.M. Deng, Phys. Dark Univ. 30, 100629 (2020). https://doi.org/ 10.1016/j.dark.2020.100629

175. H.Y. Lin, X.M. Deng, Phys. Dark Univ. 31, 100745 (2021). https:// doi.org/10.1016/j.dark.2020.100745 